\title{
Role of Megakaryoblastic Acute Leukemia-1 in ERK1/2- Dependent Stimulation of Serum Response Factor-Driven Transcription by BDNF or Increased Synaptic Activity
}

\author{
Katarzyna Kalita, ${ }^{1}$ Giorgi Kharebava, ${ }^{1}$ Jing-Juan Zheng, ${ }^{1}$ and Michal Hetman ${ }^{1,2}$ \\ ${ }^{1}$ Kentucky Spinal Cord Injury Research Center and Department of Neurological Surgery and ${ }^{2}$ Department of Pharmacology and Toxicology, University of \\ Louisville, Louisville, Kentucky 40292
}

\begin{abstract}
Serum response factor (SRF)-mediated transcription contributes to developmental and adult brain plasticity. Therefore, we investigated the role of a newly identified SRF coactivator, MKL1, in the regulation of SRF-driven transcription in rat forebrain neurons. MKL1 expression was found in newborn rat cortical or hippocampal neurons in culture as well as in adult rat forebrain. Immunostaining demonstrated constitutive nuclear localization of MKL1 in the CA1 region of the hippocampus, in the deep layers of the neocortex, and in cultured neurons. Overexpression of MKL1 in primary cortical neurons elevated SRF-driven transcription and enhanced its stimulation by BDNF. In addition, inhibition of endogenous MKL1 by overexpression of a dominant-negative MKL1 mutant or by small interfering RNA reduced BDNF activation of SRF-driven transcription. In neurons, endogenous MKL1 was associated with SRF-regulated chromatin regions of several endogenous genes including c-fos, JunB, Srf, and Cyr61. BDNF activation of MKL1/SRF-driven transcription was dependent on the extracellular signal-regulated kinase 1/2 (ERK1/2) pathway, which also led to MKL1 phosphorylation. Finally, synaptic activity stimulation of SRF-driven transcription was reduced by inhibition of endogenous MKL1. Conversely, synaptic activity enhanced transcription by overexpressed MKL1. MKL1 regulation by synaptic activity was mediated through the NMDA receptor-activated ERK1/2. These results suggest that neuronal MKL1 contributes to SRF-regulated gene expression induced by BDNF or synaptic activity. In addition, MKL1 appears as a novel mediator of the signaling between ERK1/2 and SRF. Moreover, MKL1 is a likely regulator of SRF-driven transcription programs that underlie neuronal plasticity.
\end{abstract}

Key words: Elk-1; MAL; MRTF-A; plasticity; MAP kinase; immediate-early genes

\section{Introduction}

Regulation of gene transcription is an integral part of the adaptive plasticity in neurons (Kaczmarek and Chaudhuri, 1997). For instance, a transcription factor, cAMP response element-binding protein (CREB), plays an important role in synaptic long-term potentiation and memory formation (Lonze and Ginty, 2002). Involvement of other transcription factors in neuronal plasticity is less well established.

Serum response factor (SRF) is a transcription factor binding to a consensus sequence known as a CArG box that is found in the promoters of several muscle-specific and immediate-early genes (IEGs), including c-fos (for review, see Miano, 2003). Conditional deletion of $S r f$ in the mouse brain resulted in impaired IEG

Received March 16, 2006; revised Aug. 18, 2006; accepted Aug. 21, 2006.

This work was supported by National Institutes of Health Grants NS047341-01 and RR015576-06 (M.H.) and by The Commonwealth of Kentucky Challenge for Excellence (M.H.), Norton Healthcare (M.H.), and the NATO Science Program (Grant LST.CLG980495 to M.H.). We thank Drs. Scott R. Whittemore, Theo Hagg, and Azad Boni for critical reading of this manuscript. Drs. Richard Treisman, Ron Prywes, Jane Cavanaugh, Jacek Jaworski, and Scott R. Whittemore provided reagents and cell lines used in this study. Drs. Theo Hagg and Peng Yang donated rat and mouse brain sections.

Correspondence should be addressed to Dr. Michal Hetman, Kentucky Spinal Cord Injury Research Center, University of Louisville, 511 South Floyd Street, MDR616, Louisville, KY 40292. E-mail: michal.hetman@louisville.edu. DOI:10.1523/JNEUROSCI.2644-06.2006

Copyright $\odot 2006$ Society for Neuroscience $\quad$ 0270-6474/06/2610020-13\$15.00/0 induction by neuronal activity, deficiencies in hippocampal synaptic plasticity, and reduced learning (Ramanan et al., 2005; Etkin et al., 2006). In addition, impaired axonal outgrowth, guidance, and synaptic targeting were also reported (Knoll et al., 2006). These observations suggest that SRF controls gene expression programs that are critical for neuronal development and adult brain plasticity.

Increased synaptic activity and neurotrophins, including BDNF, provide signals that trigger neuronal plasticity. The extracellular signal-regulated kinase $1 / 2(\mathrm{ERK} 1 / 2)$ pathway is a critical pro-plasticity transducer for these signals (Impey et al., 1999; Adams and Sweatt, 2002) and is also required for plasticityassociated expression of IEGs (Sgambato et al., 1998b; Valjent et al., 2001). ERK1/2 can regulate SRF-driven transcription by phosphorylation of p62TCF/Elk-1, a member of a ternary complex factor (TCF) family, the binding sites of which flank CArG boxes in several IEG promoters (Shaw and Saxton, 2003). Although Elk-1 is expressed in CNS neurons and is phosphorylated by neuronal activity-stimulated ERK1/2 (Sgambato et al., 1998a; Vanhoutte et al., 1999), its deficiency does not abolish IEG induction by neuronal activity (Cesari et al., 2004). Also, inhibition of SRF-DNA but not Elk-1-DNA binding resulted in deficits in long-term memory (Dash et al., 2005). Therefore, in neurons, there may be alternative mechanisms linking plasticity- 
associated signaling pathways including ERK1/2 to SRF-driven transcription.

In addition to TCF/Elk-1, SRF can be regulated by myocardin and myocardin-related transcription factors including megakaryoblastic acute leukemia-1 (MKL1/MAL/MRTF-A/BSCA) (for review, see Cen et al., 2004; Wang and Olson, 2004). This regulation is independent of TCF/Elk-1 and contributes to muscle-specific gene expression as well as SRF activation by a small GTPase, RhoA. MKL1 activates SRF by binding to it. This is facilitated if SRF interacts with DNA and may require MKL1 contacting an unknown DNA sequence flanking the CArG box (Zaromytidou et al., 2006). In fibroblasts, MKL1 induced several genes including neuronal activity-regulated Srf or JunB (Kaczmarek and Chaudhuri, 1997; Morris et al., 1999; Selvaraj and Prywes, 2004). MKL1 activation was reported in embryonic neurons that expressed mutant proteins stimulating the RhoA pathway (Tabuchi et al., 2005). However, it is unclear whether MKL1 contributes to activation of SRF-driven transcription by physiological stimuli regulating brain development and/or synaptic plasticity.

This study was aimed at determining whether MKL1 is a partner of SRF in BDNF- or synaptic activity-stimulated transcription in forebrain neurons. Also, we investigated the role of ERK1/2 in MKL1 regulation by these stimuli.

\section{Materials and Methods}

Materials. The following plasmids have been described previously: wildtype flag-MKL1, dominant-negative flag-MKL1 (C630), 1xSRE, pm18, and c-fos enhancer (Wang and Prywes, 2000; Cen et al., 2004); Gal4 and Gal4-SRF (Hanlon and Sealy, 1999); Gal4-E1B-luc (Impey et al., 1998); EF1 $\alpha$ LacZ; CRE-Luc (CRE-luciferase reporter) (Impey et al., 1998); hemagglutinin (HA)-tagged expression vectors for constitutive active MKK1 (Mansour et al., 1994); HA-tagged expression vectors for wildtype ERK1 and ERK2 (Frost et al., 1997), constitutive active MEK5 and wild-type ERK5 (Cavanaugh et al., 2001), and constitutive active phosphatidylinositol-3 kinase (PI3K) (p110*) (Hu et al., 1995); pON260 (Cherrington and Mocarski, 1989); Aktwt (Dudek et al., 1997); and pSUPER vector (Brummelkamp et al., 2002). Small interfering RNA (siRNA) constructs that were based on the pSUPER siRNA expression vector and targeting green fluorescent protein (GFP) were donated by Dr. J. Jaworski (International Institute of Molecular and Cell Biology, Warsaw, Poland). 5xSRE and 5xSRF reporters were purchased from Stratagene (La Jolla, CA). The following antibodies and reagents were obtained from commercial sources: rabbit and goat anti-MRTF-A (MKL1) antibodies (C-19, H-140; Santa Cruz Biotechnology, Santa Cruz, CA); rabbit anti-phospho-ERK1/2 antibody [pThr183/pTyr185, anti-active MAPK (mitogen-activated protein kinase); Promega, Madison, WI]; mouse anti-ERK1/2 antibody (Cell Signaling Technology, Beverly, MA); mouse anti- $\beta$-galactosidase ( $\beta$-gal; Promega); mouse antiflag (M2 monoclonal; Sigma, St. Louis, MO); anti-SRF (G-20; Santa Cruz Biotechnology); anti-JunB (C-11; Santa Cruz Biotechnology); mouse microtubule-associated protein 2 (MAP2) (Sigma); $\beta$-actin (Sigma); anti-glyceraldehyde-3-phosphate dehydrogenase (GAPDH; Chemicon, Temecula, CA); Alexa 488- and Alexa 555-conjugated secondary antibodies (Invitrogen, Carlsbad, CA); recombinant human active ERK2 (Upstate Biotechnology, Lake Placid, NY); and BDNF, MK-801 (dizocilpine maleate), NMDA, $\mathrm{KCl}$, tetradotoxin (TTX), 4-aminopyridine (4-AP), LY294002 [2-(4-morpholinyl)-8-phenyl-1(4H)-benzopyran-4-one], U0126 [1,4-diamino-2,3-dicyano-1,4-bis(2-aminophenylthio)butadiene], Y27632 [(R)-(t)-trans- $N$-(4-pyridyl)-4-(1-aminoethyl)-cyclohexanecarboxamide, $2 \mathrm{HCl}$ ], bicuculline, and 6-cyano-7-nitroquinoxaline-2,3-dione (Sigma, or Calbiochem, La Jolla, CA).

Generation of siRNA expression constructs. To generate MKL1 siRNA constructs, the rat MKL1 mRNA (XM 235497) sequence was analyzed using siRNA design software (http://sonnhammer.cgb.ki.SE/). Two sequences corresponding to nucleotides $732-750$ or $1242-1260$, respectively, were selected. Comparison of mouse, rat, and human mRNA se- quences of MKL1 demonstrated that the two sequences were completely conserved between rodents and humans. Oligonucleotides were designed (sequence 1, gatccccccaagcagctgaagctgaattcaagagattcagcttcagctgcttggtttttggaaa; sequence 2, gatccccccaaggagctgaagccaaattcaagagatttggcttcagctccttggtttttggaaa) together with their complementary counterparts, annealed, and subcloned into pSUPER vector digested with BglII and HindIII (Brummelkamp et al., 2002). Elk-1 siRNA (siElk-1) constructs were designed against rat Elk-1 mRNA (XM234523) corresponding to nucleotides 332-350 or 1203-1221, respectively, and cloned into pSUPER vector. The sequences of oligonucleotides used for the siElk-1 generation were as follows: sequence 1 , gatcccccgcaagaacaagaccaacattcaagagatgttggtcttgttcttgcgttttggaaa; sequence 2, gatccccgaacaagctctggtcttcattcaagagatgaagaccagagcttgttctttttggaaa.

Cell culture and transfection. COS-7 cells were cultured in DMEM (Sigma) supplemented with 10\% FBS (Invitrogen), $100 \mathrm{U} / \mathrm{ml}$ penicillin, and $0.1 \mathrm{mg} / \mathrm{ml}$ streptomycin. Cortical or hippocampal neurons were prepared from newborn Sprague Dawley rats at postnatal day 0 as described previously (Habas et al., 2006). Briefly, the culture medium was basal medium Eagle supplemented with 10\% heat-inactivated bovine calf serum (Hyclone, Logan, UT), $35 \mathrm{~mm}$ glucose, $1 \mathrm{~mm}$ L-glutamine, 100 $\mathrm{U} / \mathrm{ml}$ penicillin, and $0.1 \mathrm{mg} / \mathrm{ml}$ streptomycin. Cytosine arabinoside $(2.5$ $\mu \mathrm{M})$ was added to cultures on the second day after seeding [ $2 \mathrm{~d}$ in vitro (DIV)] to inhibit the proliferation of non-neuronal cells. Cells were used for experiments at 6-7 DIV unless indicated otherwise. Transient transfections were performed on 4 or 5 DIV using the Lipofectamine2000 reagent (Invitrogen) as described previously (Hetman et al., 2002). Electroporation of freshly dissociated newborn rat cortical neurons was conducted using the rat neuron nucleofection reagents (Amaxa, Köln, Germany). COS-7 cells were transfected with $4 \mu \mathrm{g}$ of plasmid DNA per 60 $\mathrm{mm}$ plate using Lipofectamine2000 following the manufacturer's recommendations (Invitrogen).

Drug treatment. BDNF was diluted in PBS containing $0.1 \%$ bovine serum albumin before addition to the cells. U0126, LY294002, and Y27632 were dissolved in dimethylsulfoxide (DMSO), whereas NMDA, $\mathrm{KCl}$, and bicuculline/4-AP were dissolved in culture media. The final concentration of DMSO in the medium was $0.2-0.4 \%$. All inhibitors were added $30 \mathrm{~min}$ before stimulation in cotreatment experiments. Bicuculline/4-AP treatments were done at 7 DIV.

$R N A$ isolation and reverse transcription-PCR. RNA was isolated from $5 \times 10^{6}$ cells or from newborn rat tissues using TRI reagent (Sigma). The remaining DNA was removed by digestion with DNase I (Promega), and RNA was reverse transcribed with the AMV First-Strand cDNA Synthesis kit (Invitrogen) in the presence of oligo-dT. The cDNA was amplified by PCR with a set of oligonucleotides designed to recognize mRNAs for MKL1 (5' cactcatcaagcaaagccaaccga ${ }^{\prime} ; 5^{\prime}$ aacttcagctcctgcttcagctct3'), SRF (5' acacgactttcagcaagaggaaga $3^{\prime} ; 5^{\prime}$ gttggtgactgtgaatgctggctt $3^{\prime}$ ), and myocardin $\left(5^{\prime}\right.$ tcctttgaacaggcatcttcggga ${ }^{\prime} ; 5^{\prime}$ actgaagagatcttccgcagcctt $\left.3^{\prime}\right)$. The following temperature profile was applied for both reactions: $94^{\circ} \mathrm{C}$ for $30 \mathrm{~s}, 55^{\circ} \mathrm{C}$ for $30 \mathrm{~s}, 72^{\circ} \mathrm{C}$ for $1 \mathrm{~min}$, followed by a $15 \mathrm{~min}$ incubation at $72^{\circ} \mathrm{C}$. Amplification of $18 \mathrm{~S}$ RNA cDNA was used for verification of the amount of template used for the reactions $\left(5^{\prime}\right.$ cgcggttctatttgttggt $3^{\prime}$; $5^{\prime}$ agtcggcatcgtttatggtc $3^{\prime}$ ).

Immunohistochemistry. Naive young adult female Sprague Dawley rats ( $n=3$; 2-3 months old; weight, 180-220 g; Harlan, Indianapolis, IN) were housed in groups under a $12 \mathrm{~h}$ light/dark cycle and with ad libitum access to water and food. All animals were treated in accordance with the guidelines of the National Institutes of Health Guide for the Care and Use of Laboratory Animals and the University of Louisville Guidelines for the Care and Use of Laboratory Animals. Anesthetized animals were perfused transcardially, and free-floating $30-\mu \mathrm{m}$-thick coronal brain sections were prepared after postfixation (Yang et al., 2005). For staining, sections were washed in Tris-buffered saline (TBS; $0.05 \mathrm{~mm}$ Tris and $0.9 \% \mathrm{NaCl}$, $\mathrm{pH} 8.0$ ), the endogenous peroxidase was quenched for $5 \mathrm{~min}$ (10\% methanol and $1 \% \mathrm{H}_{2} \mathrm{O}_{2}$ in TBS), and the cell membrane was permeabilized (15 min in $0.2 \%$ Triton X-100 in TBS). A goat polyclonal anti-MKL1 antibody (anti-MRTF-A, C19; 1:100; Santa Cruz Biotechnology) was applied in a blocking buffer (10\% normal goat serum in TBS overnight at $\left.4^{\circ} \mathrm{C}\right)$. The sections were then washed with TBS and incubated with the secondary antibody (1:250, biotinylated anti-goat IgG antibody, $2 \mathrm{~h}$ at 
room temperature; Vector Laboratories), followed by a standard $\mathrm{ABC} /$ DAB histochemistry (Vector Laboratories).

Immunocytochemistry. Immunostaining for $\beta$-gal and flag epitope was performed as described previously (Hetman et al., 1999). For staining of endogenous proteins, neurons were fixed with ice-cold $4 \%$ paraformaldehyde in PBS for 20 min. After fixation, cells were incubated in Tris-A buffer $(250 \mathrm{~mm} \mathrm{NaCl}, 0.1 \mathrm{~m}$ Tris-HCl, $\mathrm{pH} 7.5$, and $0.1 \%$ Triton X-100) for $15 \mathrm{~min}$ at room temperature, then incubated with primary antibodies in Tris-B buffer (Tris-A buffer, 2\% BSA, and 1.5\% normal horse serum) overnight at $4^{\circ} \mathrm{C}$, followed by a standard immunocytochemistry protocol. Primary antibodies were mouse monoclonal anti-MAP2 (1:400; Sigma) and rabbit polyclonal anti-MRTF-A (1:100, H140; Santa Cruz Biotechnology). Secondary antibodies conjugated to Alexa 488 and Alexa 555 (1:100; Invitrogen) were used for double labeling.

Western analysis. Western blot was performed using a standard procedure (Hetman et al., 1999). The primary antibodies were as follows: MRTF-A (C19, 1:500; Santa Cruz Biotechnology); pERK1/2 (1:2000; Promega), ERK1/2 (1:1000; Cell Signaling Technology), $\beta$-actin (1:100; Sigma), flag (1:1000; Sigma), SRF (1:500; Santa Cruz Biotechnology), JunB (1:500; Santa Cruz Biotechnology), and GAPDH (1:500; Chemicon). To detect the phosphorylation shift of MKL1 by Western blotting, $6 \%$ polyacrylamide gels were used. For all other epitopes, proteins were separated on $10 \%$ gels.

Luciferase reporter gene assays. Neurons cultured on 24 -well plates $(5 \times$ 105 /well) were transfected with Lipofectamine2000. Luciferase and $\beta$-gal activities were assayed using commercial assay kits (Promega). Transcriptional activity was determined as a luciferase activity normalized to $\beta$-gal activity and compared with unstimulated controls.

Chromatin immunoprecipitation assay. Chromatin immunoprecipitation (ChIP) assays were performed using the ChIP assay kit (Upstate Biotechnology) following the manufacturer's protocol with the following modifications. Neurons $\left(1 \times 10^{7}\right.$ cells/sample $)$ were fixed with $1 \%$ formaldehyde at room temperature for $20 \mathrm{~min}$. The chromatin was sonicated 10 times for $10 \mathrm{~s}$ each with a $20 \mathrm{~s}$ interpulse interval using a $150 \mathrm{~W}$ Sonifier Cell Disrupter 185 (Branson, Danbury, CT) at 30\% power setting. For immunoprecipitation, one-half of the sample $\left(\sim 5 \times 10^{6}\right.$ cells/ $0.2 \mathrm{ml}$ ) was diluted 10-fold with ChIP dilution buffer (Upstate Biotechnology) and incubated with $10 \mu \mathrm{g}$ of anti-SRF polyclonal rabbit antibody (G-20; Santa Cruz Biotechnology), anti-MKL1 rabbit serum (a gift from Dr. R. Treisman, London Research Institute, London, UK), or $10 \mu \mathrm{g}$ of MKL1 polyclonal rabbit antibody (MRTF-A, H-140; Santa Cruz Biotechnology) at $4^{\circ} \mathrm{C}$ overnight. Immune complexes were collected with protein A-agarose (Upstate Biotechnology). For PCR, specific sets of primers were designed to amplify DNA flanking active CArG boxes from several genes including c-fos (position - 307; forward, 5 'tcccccttgcgctgcaccctcaga 3'; reverse, $5^{\prime}$ caacagggaccggccgtggaaacct $3^{\prime}$ ), JunB (position + 2084; forward, 5' aacccggaatgtgcaagcacgg $3^{\prime}$, reverse: 5' aggctggagtccgtgaattggatt3'), Srf (2 CArG boxes at positions -43 and -63 , respectively; SRF forward, 5' ggtgccggaagcgcggaccaat3'; SRF reverse, 5' tgcggccgtcagcgtccc3'), and Cyr61 (position - 1920; forward, $5^{\prime}$ ccaacgaatttcctgctctggg $3^{\prime}$; reverse, 5' agaatggagaggagggttcctgg $3^{\prime}$ ). Primers amplifying the promoter region of the rat Gapdh gene were used as a negative control (forward, 5' aaacaagttcaccaccatgtgaaa $3^{\prime}$; reverse, 5' ccagggattgaccaaaggtgagtt $\left.3^{\prime}\right)$. Immunoprecipitated chromatin was aliquoted and used for PCRs, the conditions of which were determined empirically to ensure amplification in the linear range (details can be provided by the authors on request). PCR products were separated by $2 \%$ agarose gel electrophoresis and visualized with ethidium bromide.

MKL1 phosphorylation in vitro. COS-7 cells were lysed in Triton lysis buffer [20 mm Tris- $\mathrm{HCl}$, pH 7.4, $150 \mathrm{~mm} \mathrm{NaCl}, 25 \mathrm{~mm} \beta$-glycerolphosphate, 2 mм EDTA, 2 mм NaPPi, 1 mм $\mathrm{Na}_{3} \mathrm{VO}_{4}$, $1 \%$ Triton X-100, $10 \%$ glycerol, 1 mm DTT, and protease inhibitors (set III; Calbiochem)] for $10 \mathrm{~min}$ and scraped, and the lysate was centrifuged at $14,000 \times \mathrm{g}$. MKL1 was immunoprecipitated using a standard protocol with protein $\mathrm{G}$ beads coupled to $10 \mu \mathrm{g}$ of anti-flag antibody per $60 \mathrm{~mm}$ plate. Anti-flag immunoprecipitates from empty vector-transfected COS-7 cells were used as a negative control. Phosphorylation reactions were performed in $20 \mu \mathrm{l}$ of kinase buffer ( $25 \mathrm{~mm}$ HEPES, pH 7.4, 25 mм $\beta$-glycerolphosphate, $25 \mathrm{mM} \mathrm{MgCl}_{2}$, and $1 \mathrm{mM} \mathrm{Na}_{3} \mathrm{VO}_{4}$ ) containing $50 \mu \mathrm{M}$ ATP and 0.2
A

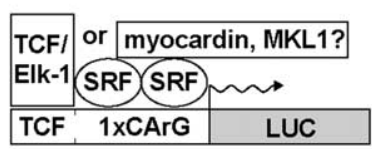

1XSRE

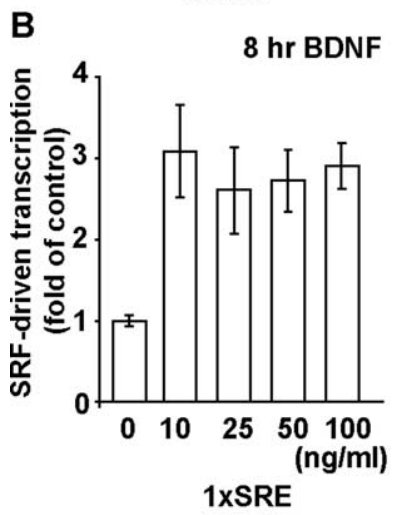

D BDNF 10ng/ml

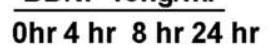

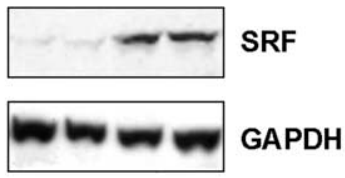

$\begin{array}{llll}1 & 1 & 6.6 & 4.9\end{array}$

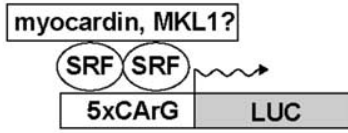

$5 \times$ SRF

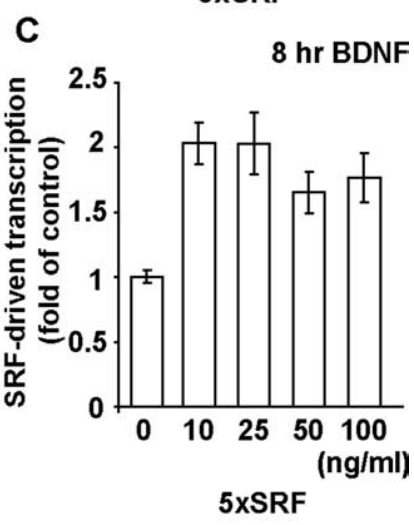

E

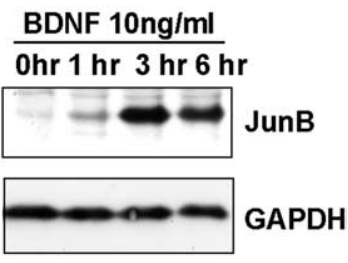

$1 \quad 4.611 .38 .2$
Figure 1. BDNF activates SRF-driven transcription independently of TCF/Elk-1. $A$, The diagrams of 1 XSRE and 5 XSRF luciferase (LUC) reporter plasmids. The binding sites for the transcription factors SRF (CArG box) and TCF/Elk-1 (TCF) are indicated. B, C, At 4 DIV, cortical neurons were cotransfected with EF1 $\alpha$ lacZ $\beta$-gal expression plasmid together with 1XSRE or 5XSRF reporters $\left(0.1+0.1 \mu \mathrm{g}\right.$ of plasmid DNA $/ 5 \times 10^{5}$ cells). Two days after transfection, cells were stimulated with BDNF as indicated. SRF-driven transcription was estimated by determining the luciferase/ $\beta$-gal activity ratio and is presented as the fold of unstimulated controls. The data represent quadruplicate determinations. Similar results were obtained in another experiment. Error bars indicate SEM. D, E, At 6 DIV, Cortical neurons were stimulated with BDNF. For SRF expression studies, BDNF stimulations were performed in the presence of TTX (1 $\mu \mathrm{M})$ and CNQX (40 $\mu \mathrm{m}$; 6-cyano-7-nitroquinoxaline-2,3-dione), which were added $12 \mathrm{~h}$ before the neurotrophin. The numbers under the blots indicate relative levels of SRF or JunB after normalization against GAPDH, which was detected by reprobing the blots with an anti-GAPDH antibody. Note the increased levels of SRF or JunB in neurons stimulated with BDNF for 8 and $24 \mathrm{~h}$ or 1,3 , and $6 \mathrm{~h}$, respectively. Because expression of SRF and JunB is stimulated by cooperation between SRF and myocardin family members, these data suggest their activation in BDNF-treated neurons.

$\mu \mathrm{g}$ of recombinant active ERK2 per sample at $30^{\circ} \mathrm{C}$ for $15 \mathrm{~min}$. Fifty microcuries of $\left[\gamma^{32} \mathrm{P}\right]$ ATP per sample were included for radioactive labeling of phospho-MKL1. The reactions were stopped by the addition of $1 \times$ SDS-PAGE loading buffer, boiled for $5 \mathrm{~min}$, and analyzed by SDS-PAGE, followed by autoradiography or Western blotting with antiMKL1 antibody.

Statistical analysis. Statistical analysis of the data were performed using one-way ANOVA.

\section{Results}

\section{BDNF activates SRF-driven transcription in the absence of a} TCF binding site

To determine whether SRF-driven transcription in neurons might be regulated by mechanisms other than those operated by TCF/Elk-1, we compared transcriptional activity of two reporter constructs, $1 x$ SRE and 5xSRF. The reporter plasmid 1xSRE contains the CArG box from the c-fos promoter that is preceded by a TCF binding sequence (Fig. $1 A$ ). In contrast, the plasmid 5xSRF has five CArG boxes without any TCF sites (Fig. $1 A$ ). Thus, SRF- 
A

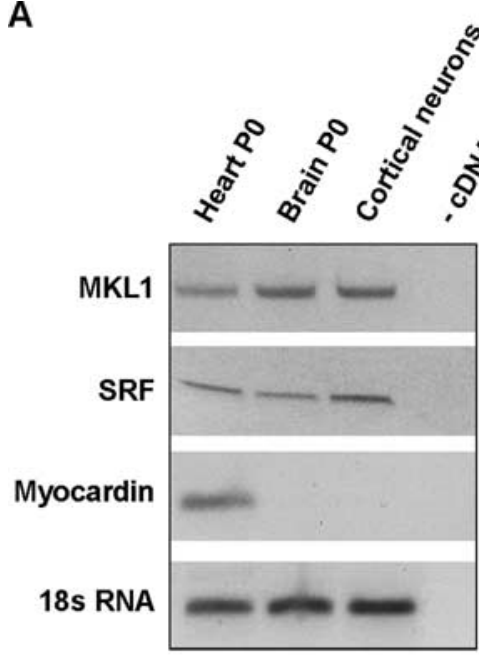

RT-PCR

B

$\beta$-actin

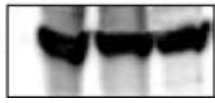

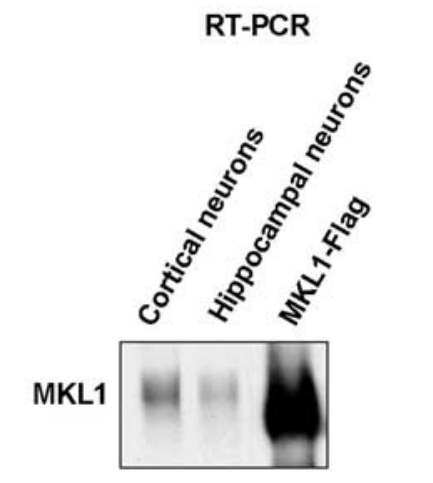

C

cortical neurons hippocampal neurons
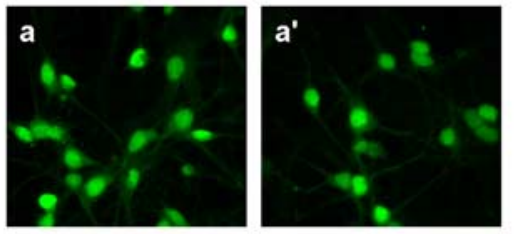

b

MAP-2
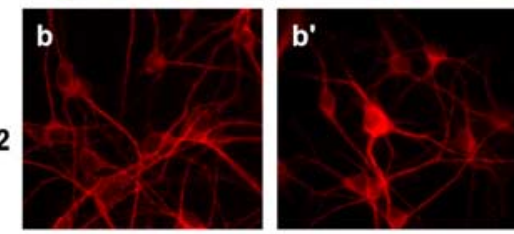

c

Hoechst

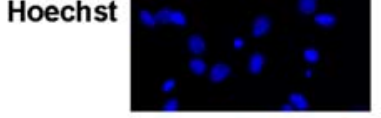

$c^{\prime}$

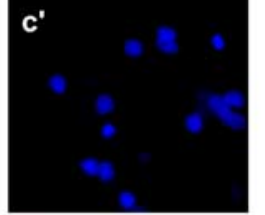

MKL1/

MAP-2I Hoechst
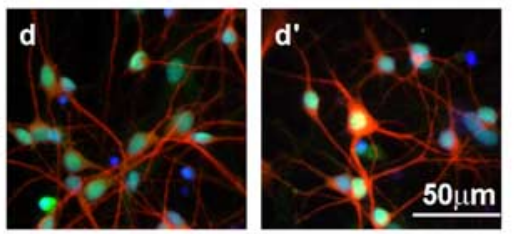

gene is strongly regulated by MKL1 (Selvaraj and Prywes, 2004). Together, these observations support a possibility that in primary cortical neurons there are TCF/ Elk-1-independent mechanisms that regulate SRF-driven transcription and that these mechanisms are engaged, at least in part, in response to BDNF.

\section{MKL1 expression in forebrain neurons} To determine whether a transcriptional coactivator, MKL1, may provide an alternative control over SRF in CNS neurons, we first determined whether it is expressed in primary cortical or hippocampal neurons from newborn rats. At 6 DIV, both MKL1 and SRF mRNAs were detected in primary cortical neuron cultures (Fig. $2 A$ ). They were also present in the rat newborn brain (Fig. $2 A$ ). In contrast, mRNA encoding a muscle- specific relative of MKL1, myocardin, was found only in the heart (Fig. 2A). Using Western blot analysis, we confirmed the expression of MKL1 protein in both cortical and hippocampal neuron cultures (Fig. 2B). The lower electrophoretic mobility of endogenous rat MKL1 compared with overexpressed human flag-tagged MKL1 may be attributable to differences in polypeptide chain lengths and/or posttranslational modifications. Double immunofluorescence for MKL1 and a neuron-specific marker, MAP2, demonstrated that neurons are the primary site of MKL1 expression in these cultures (Fig. 2C). MKL1 was observed in nuclei but not in perikarial cytosol or neurites (Fig. 2C). In addition, there were few MAP2-negative cells demonstrating MKL1 presence in the nuclei (Fig. 2C). This suggests that some non-neuronal cells express MKL1.

We also studied MKL1 protein expresdriven transcription detected using 1xSRE may be TCF dependent or independent, whereas 5xSRF would reveal only TCFindependent SRF activity. In cultured cortical neurons that were stimulated at 6 DIV with $10 \mathrm{ng} / \mathrm{ml}$ BDNF for $8 \mathrm{~h}$, an increase in 1xSRE or 5xSRF reporter activities was observed (3.1- or 2-fold of controls, respectively) (Fig. $1 B, C$ ). Similar increases were also found with the $5 \times$ SRE reporter construct (data not shown). This suggests that BDNF activates SRF-driven transcription that is independent of TCF/Elk-1.

To investigate whether BDNF can increase expression of genes with a transcription that is under regulation of SRF but not TCF/ SRF, we studied the effects of BDNF on SRF protein levels in cortical neurons. The Srf gene promoter region has a CArG box but no adjacent TCF binding sites and is strongly upregulated in response to SRF activation by myocardin family members including MKL1 (Spencer and Misra, 1996; Miano, 2003; Selvaraj and Prywes, 2004). Increased levels of SRF (67 kDa isoform) were found at 8 or $24 \mathrm{~h}$ after treatment with $10 \mathrm{ng} / \mathrm{ml}$ BDNF (Fig. $1 D$ ). In addition, BDNF induced expression of JunB that reached maximal levels $3 \mathrm{~h}$ after treatment (Fig. $1 E$ ). Similarly to $\operatorname{Srf}$, JunB sion in the forebrain of adult rodents. In rats, MKL1 immunoreactivity was detected at high levels in deep layers of the neocortex (Fig. $3 a-a^{\prime \prime}$ ) and in the hippocampal pyramidal cell layer of the CA1 subfield (Fig. $3 b-b^{\prime \prime}$ ). In these locations, the pattern of MKL1 staining indicated its nuclear localization (Fig. $3 a^{\prime \prime}, b^{\prime \prime}$ ). The specificity of MKL1 labeling was confirmed in control experiments, in which the primary antibody was replaced by the nonimmune goat serum (data not shown). Also, a similar MKL1 expression pattern was noted in the forebrains of adult mice (data not shown). Thus, MKL1 is expressed in cortical or hippocampal neurons both in culture and in the adult brain. Therefore, MKL1 is a good candidate regulator of SRF-driven transcription in these CNS cells.

\section{Overexpression of MKL1 activates SRF-driven transcription in neurons}

As a first step to evaluate whether MKL1 contributes to the regulation of neuronal SRF activity, we analyzed the effects of overexpressed flag-tagged MKL1 on SRF-driven transcription in primary cortical neurons. Two days after transfection of the MKL1 
expression plasmid, increased SRF transcriptional activity was detected using 1xSRE or 5xSRF reporters (Fig. $4 A, B$ ). The increases were 6.7- or 14.9-fold of empty vector controls, respectively $(p<$ 0.001 ) (Fig. $4 A, B$ ). In addition, the overexpressed MKL1 strongly enhanced SRF transcriptional activation in response to $8 \mathrm{~h}$ stimulation with $10 \mathrm{ng} / \mathrm{ml}$ BDNF (Fig. $4 A, B)$. Although in empty vectortransfected cells BDNF increased 1xSRE activity to 3.1-fold of unstimulated controls, in MKL1-overexpressing neurons, the increase was 56.1-fold over these controls (Fig. 4A). Similar trends were revealed with $5 x$ SRF reporter plasmid (Fig. $4 B$ ). These data indicate that overexpression of MKL1 is able to regulate SRFdriven transcription in neurons and that it is highly regulated by BDNF.

To test whether in neurons MKL1 regulates SRF-driven transcription by increasing the transactivation potential of the SRF, we determined the effects of overexpressing flag-tagged MKL1 on the transcriptional activity of the coexpressed Gal4-SRF fusion protein. Gal4-SRF is an engineered transcription factor that contains a DNA binding domain of a yeast transcription factor, Gal4, fused to the SRF and therefore is able to activate a reporter construct with a Gal4 binding sequence. An expression plasmid for the Gal4 DNA binding domain was used as an additional control (Fig. 4C). Whereas coexpression of MKL1 and Gal4-SRF did not significantly alter the basal transcriptional activity of Gal4-SRF, the BDNF-mediated increase in Gal4-SRF-driven transcription was almost doubled in the presence of MKL1 (4.8- vs 9.4-fold of controls in vector- or MKL1transfected neurons, respectively) (Fig. 4C). Together, these data suggest that in neurons, MKL1 is a coactivator of SRF and that it may provide a link between neurotrophin signaling and SRFdriven transcription.

\section{Endogenous MKL1 contributes to BDNF stimulation of SRF-driven transcription}

To test whether endogenous MKL1 is required for BDNF stimulation of SRF-driven transcription, cortical neurons were transfected with an expression vector for a flag-tagged dominant-negative (C630) mutant of MKL1 (DN-MKL1) (Cen et al., 2003). This mutant was constructed by deleting a cDNA region that encodes for the C-terminal portion of the protein starting at amino acid residue 630. The deletion eliminated the putative transactivation domain resulting in a protein that interacted with SRF blocking its regulation by the wild-type MKL1. Using flag/ $\beta$-gal ( $\beta$-gal) double immunocytochemistry, we verified that the DN-MKL1 was expressed in transfected neurons. Forty-eight hours after transfection, flag-DN-MKL1 was detected in neurons that showed normal morphology, indicating that it does not induce acute cytotoxicity (Fig. $5 A$ ). Counterstaining with Hoechst 33258 demonstrated that most of the flag-DN-MKL1 was in cell nuclei (Fig. 5A).

To test whether DN-MKL1 is able to inhibit MKL1/SRF- driven transcription in neurons, we examined its effects on the BDNF activation of overexpressed wild-type MKL1. DN-MKL1 abolished BDNF stimulation of MKL1/SRF-driven transcription in neurons that overexpressed wild-type MKL1 (Fig. 5B). These data demonstrate that in neurons, DN-MKL1 is a strong inhibitor of MKL1/SRF-driven transcription.

To determine the contribution of endogenous MKL1 to BDNF induction of SRF-driven transcription, we studied the effects of DN-MKL1 on transcriptional activity of $1 \mathrm{xSRE}$ or $5 \mathrm{xSRF}$ reporter constructs. In both cases, DN-MKL1 significantly reduced transcriptional activation induced by $8 \mathrm{~h}$ treatment with 10 $\mathrm{ng} / \mathrm{ml} \operatorname{BDNF}(p<0.001)$ (Fig. 5C,D). In empty vectortransfected neurons, BDNF increased 1xSRE activity 2.8-fold of controls, and DN-MKL1 decreased this induction to 1.5fold of control $(p<0.001)$ (Fig. $5 C$ ). Similar trends were observed with $5 \mathrm{xSRF}$ reporter (Fig. $5 D$ ). These effects were specific to SRF because DN-MKL1 did not affect BDNF stimulation of a cAMP response element (CRE)-driven transcription (Fig. 5E).

To exclude the possibility that reductions in SRF transcription by DN-MKL1 are attributable to competitive inhibition of other SRF regulators rather than endogenous MKL1, we developed two siRNA expression plasmids to specifically knock down endogenous MKL1. A mix of these two plasmids inhibited expression of flag-MKL1 protein in cortical neurons (Fig. 6A). Flag-MKL1 lev- 

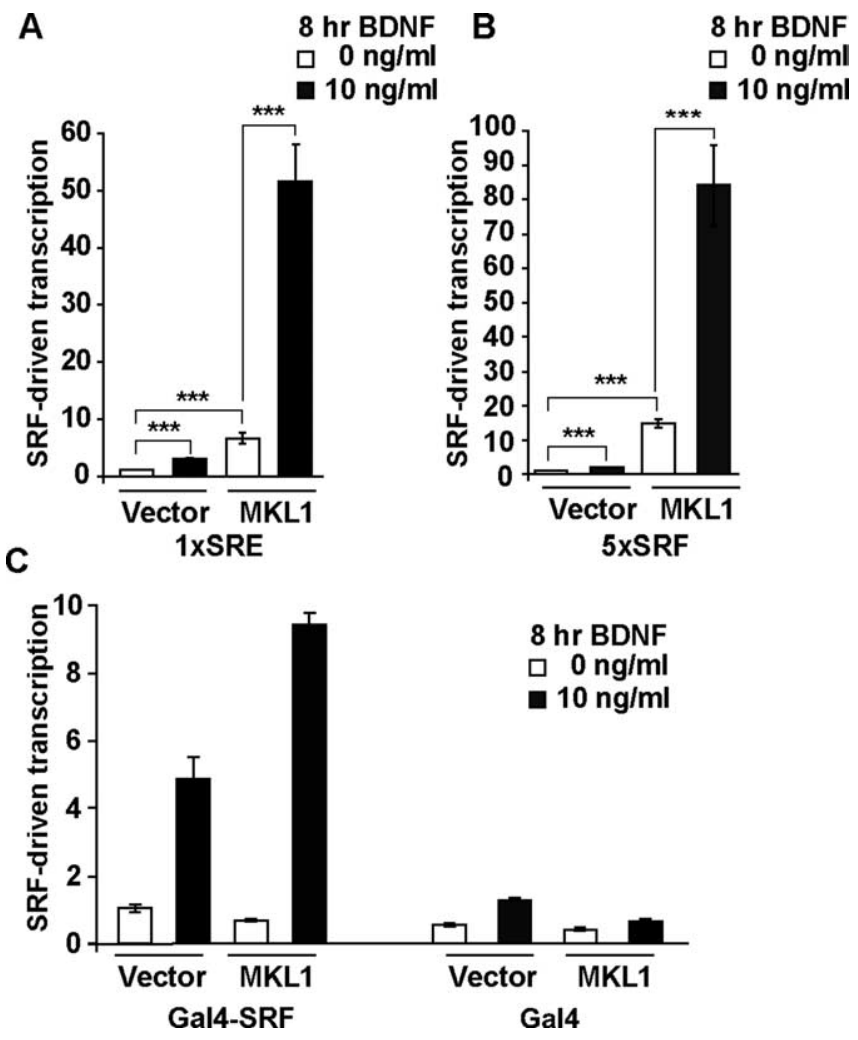

Figure 4. Overexpression of MKL1 in neurons stimulates SRF-driven transcription. $A, B$, At 4 DIV, cortical neurons were cotransfected with the indicated reporter together with EF1 $\alpha$ LacZ and either empty cloning vector (Vector) or wild-type MKL1 $(0.1+0.1+0.1 \mu \mathrm{g}$ of plasmid $\mathrm{DNA} / 5 \times 10^{5}$ cells, respectively). Forty-eight hours after transfection, cells were treated as indicated. Data represent quadruplicate determinations from four independent experiments. Error bars indicate SEM. ${ }^{* *} p<0.001$. Note a significant increase in SRF-driven transcription in MKL1-overexpressing neurons that was further potentiated by BDNF ( $p<0.001)$. C, At 4 DIV, cortical neurons were cotransfected with a Gal4-dependent luciferase reporter (E1B-luc) and the following expression plasmids: EF1 $\alpha$ LacZ, Gal4-SRF, and wild-type MKL1 $(0.033+0.1+$ $0.07+0.1 \mu \mathrm{g}$ of plasmid DNA $/ 5 \times 10^{5}$ cells, respectively). Gal4 DNA-binding domain expression plasmid (Gal4) and MKL1 empty cloning vector (Vector) were used as controls. After $48 \mathrm{~h}$, cells were stimulated as indicated. Note that MKL1 enhanced BDNF stimulation of Gal4-SRF. Data represent quadruplicate determinations. Error bars indicate SD. Similar results were obtained in four independent experiments.

els were unaffected by control siRNA plasmids, including the empty siRNA expression vector (pSUPER) or a siRNA expression construct producing a siRNA against a GFP (Fig. 6A). Moreover, in neurons that were cotransfected with MKL1 siRNA expression plasmids (siMKL1) together with wild-type MKL1, BDNF stimulation of SRF-driven transcription by the overexpressed MKL1 was reduced (Fig. $6 B$ ). These data indicate that siMKL1 is an effective tool to knock down neuronal MKL1.

To evaluate the effects of knocking down endogenous MKL1 on SRF-driven transcription, we cotransfected neurons with the siRNA plasmids and 1xSRE or 5xSRF reporters. As a control, we used an siRNA expression plasmid targeting GFP (siGFP). Fortyeight hours after transfection, neurons were stimulated with BDNF (10 ng/ml) for $8 \mathrm{~h}$. siMKL1 significantly reduced BDNF induction of SRF-driven transcription $(p<0.001)$ (Fig. 6C,D). This effect was specific, because siMKL1 did not affect BDNFmediated activation of CRE-driven transcription (Fig. 6E). Overall, in BDNF-stimulated neurons, siRNA knockdown of MKL1 resulted in similar inhibitions of SRF-driven transcription as those produced by the DN-MKL1. These data indicate that in neurons, endogenous MKL1 regulates SRF-driven transcription.
A
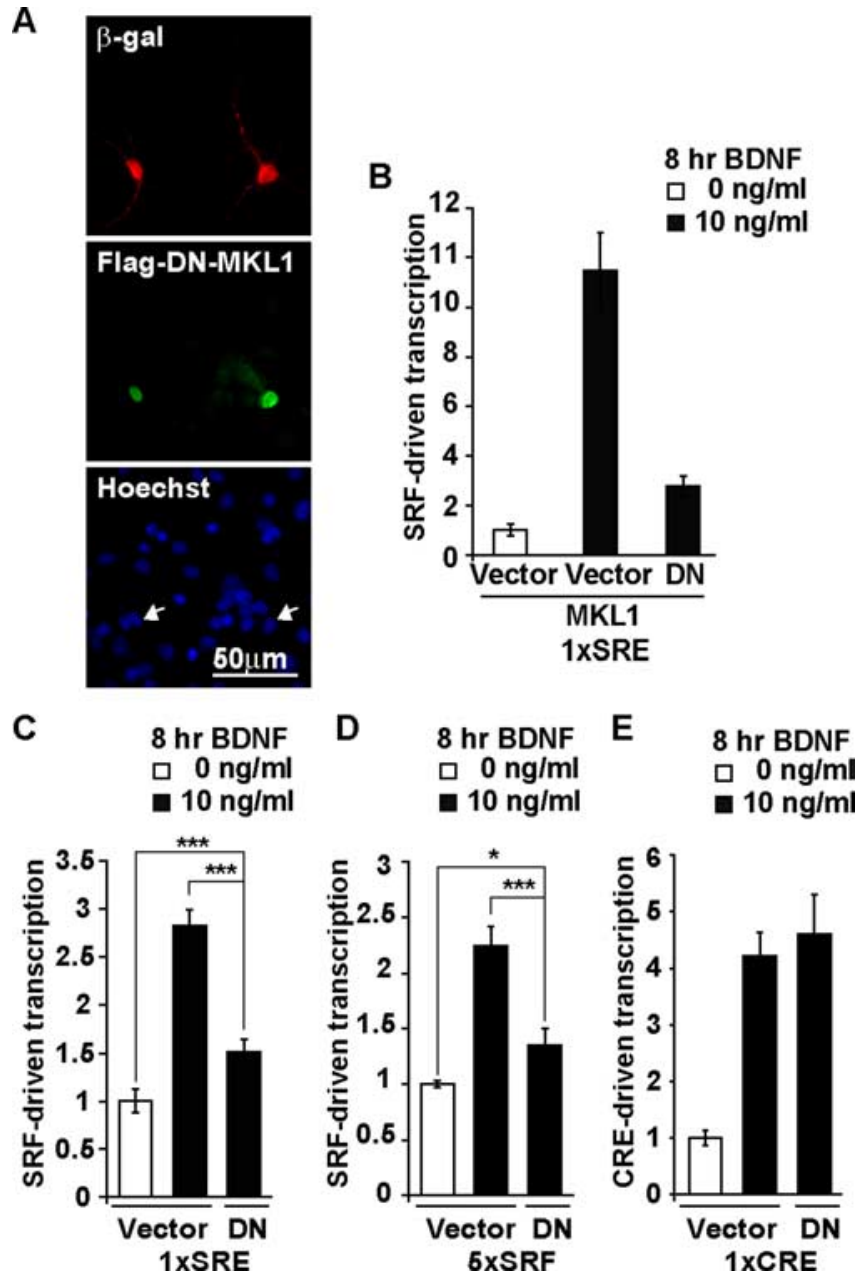

Figure 5. Inhibition of endogenous MKL1 by DN-MKL1 mutant reduces BDNF stimulation of SRF-driven transcription. At 4 DIV, cortical neurons were cotransfected with a $\beta$-gal expression plasmid [p0N260 $(\boldsymbol{A})$ or EF1 $\alpha \mathrm{LaCZ}(\boldsymbol{B}-\boldsymbol{E})$ at 0.2 or $0.1 \mu \mathrm{g}$ of plasmid DNA/5 $\times 10^{5}$ cells, respectively] together with an empty vector (Vector) or an expression construct for the flagtagged dominant-negative mutant form of MKL1 (DN-MKL1; $1 \mu \mathrm{g}$ of plasmid DNA/5 $\times 10^{5}$ cells). In $\boldsymbol{B}-\boldsymbol{E}$, cells were also transfected with the indicated transcriptional reporters ( $0.1 \mu \mathrm{g}$ of plasmid DNA $/ 5 \times 10^{5}$ cells). In $\boldsymbol{B}$, a wild-type MKL1 expression plasmid was included in the transfection mix ( $0.1 \mu \mathrm{g}$ of plasmid DNA $/ 5 \times 10^{5}$ cells). Two days after transfection, cells were either fixed and immunostained or stimulated as indicated. $\boldsymbol{A}$, Representative photomicrographs of $\beta$-gal (red) and DN-MKL1 (green) coexpressing neurons. The nuclei were visualized using Hoechst 33258 (blue). The DN-MKL1-expressing neurons displayed normal morphology, suggesting that DN-MKL1 expression did not trigger acute cytotoxicity. $\boldsymbol{B}$, Effects of DN-MKL1 on BDNF stimulation of overexpressed wild-type MKL1. Coexpression of DN-MKL1 together with MKL1 abolished MKL1-mediated activation of SRF-driven transcription in response to BDNF. C, D, Effects of DN-MKL1 on endogenous transcription machinery that mediates BDNF stimulation of SRF-driven transcription. Note that DN-MKL1 significantly reduced transcriptional activation induced by BDNF. $\boldsymbol{E}$, BDNF stimulation of $1 \times C R E$ transcriptional activity was unaffected by DN-MKL1. In $\boldsymbol{B}$ and $\boldsymbol{E}$, quadruplicate determinations from one experiment are shown. Similar results were obtained in another independent experiment. In $\boldsymbol{C}$ and $\boldsymbol{D}$, quadruplicate determinations from three independent experiments are depicted. Error bars indicate SEM; ${ }^{*} p<0.05$; ${ }^{* * *} p<0.001$.

To identify endogenous genes that may be regulated by MKL1, we performed ChIP assay using antibodies specific for SRF or MKL1. ChIP input was chromatin that was isolated from cultured cortical neurons after treatment with or without 10 $\mathrm{ng} / \mathrm{ml}$ BDNF for $30 \mathrm{~min}$. SRF or MKL1 antibodies precipitated several chromatin regions containing SRF-regulated CArG boxes (Fig. 7). These included CArG box elements from genes that were previously reported to either directly associate with MKL1 
A

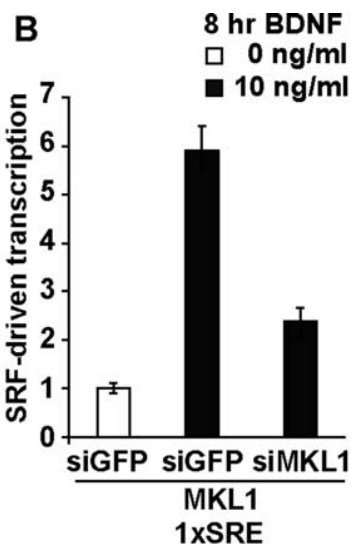

C


Figure 6. Inhibition of endogenous MKL1 by siRNA-mediated knockdown reduces BDNF stimulation of SRF-driven transcription. $A$, Cortical neurons were cotransfected by electroporation with flag-tagged MKL1 and $\beta$-gal expression constructs ( $1 \mu \mathrm{g}$ of MKL1 plus $0.2 \mu \mathrm{g}$ of p0N260 plasmid DNA/2 $\times 10^{6}$ cells) together with $1.5 \mu \mathrm{g}$ of plasmid DNA/2 $\times 10^{6}$ cells of each of the following plasmids: siRNA cloning vector (PSUPER), a 1:1 mixture of two MKL1specific siRNAs cloned in pSUPER (siMKL1), or a pSUPER-cloned siRNA targeting GFP (siGFP) that was used as additional control. Forty-eight hours after transfection, cells were lysed, and flagMKL1 levels were determined by Western blotting with an anti-flag antibody. In addition, the blot was reprobed with an anti- $\beta$-gal antibody to determine transfection efficiency. siMKL1 efficiently inhibited flag-MKL1 expression. $\boldsymbol{B}-\boldsymbol{E}$, Cortical neurons (4 DIV) were cotransfected with EF1 $\alpha$ LacZ together with the indicated reporter constructs and either a control siRNA (siGFP) or siMKL1 $\left(0.1+0.1+1.0 \mu \mathrm{g}\right.$ of plasmid DNA $/ 5 \times 10^{5}$ cells, respectively). In $\boldsymbol{B}$, neurons were additionally transfected with an expression vector for wild-type MKL1 (0.1 $\mu \mathrm{g}$ of plasmid DNA $/ 5 \times 10^{5}$ cells). Two days after transfection, cells were stimulated as indicated. $\boldsymbol{B}$, Effects of siMKL1 on BDNF stimulation of overexpressed MKL1. Coexpression of siMKL1 together with MKL1 abolished MKL1-mediated activation of SRF-driven transcription in response to BDNF. C, D, Effects of siMKL1 on endogenous transcription machinery that mediates BDNF stimulation of SRF-driven transcription. Please note that siMKL1 significantly reduced transcriptional activation induced by BDNF. $\boldsymbol{E}$, BDNF stimulation of $1 \times$ CRE transcriptional activity was unaffected by siMKL1. In $\boldsymbol{B}$ and $\boldsymbol{E}$, quadruplicate determinations from one experiment are shown. Similar results were obtained in another independent experiment. In $\boldsymbol{C}$ and $\boldsymbol{D}$, quadruplicate determinations from three independent experiments are depicted. Error bars indicate SEM. ${ }^{* * *} p<0.001$. n.S., Not significant.

(Cyr61, Srf) and/or to respond with reduced expression levels to a dominant-negative mutant form of MKL1 (c-fos, JunB, and Srf) (Cen et al., 2003; Miralles et al., 2003; Selvaraj and Prywes, 2004). The interactions between SRF or MKL1 and the chromatin were detected in either unstimulated or BDNF-stimulated neurons, indicating that in basal condition MKL1 is already associated with the chromatin (Fig. 7). Importantly, the specificity of MKL1/SRF association with the SRF-regulated regions of the chromatin is supported by the observation that neither SRF nor MKL1 antibodies were able to precipitate a 5 ' regulatory region of



Figure 7. Endogenous MKL1 is bound to the chromatin regions containing SRF-associated CArG boxes. Cultured rat cortical neurons were stimulated with $10 \mathrm{ng} / \mathrm{ml}$ BDNF for $30 \mathrm{~min}$ as indicated. Sonicated chromatin was immunoprecipitated using antibodies specific to SRF or MKL1, followed by PCR amplification of DNA sequences flanking CArG boxes in several genes, including c-fos, JunB, Srf, and Cyr61. A portion of the rat Gapdh promoter region that does not contain any CArG box was amplified to control for specificity. Sonicated chromatin without precipitation was used as a positive control (input). A precipitate obtained using chromatin from unstimulated cells and beads without antibody served as a negative control (no Ab). Similarly, no amplification was found if precipitation was performed without chromatin (Mock) or using non-immune IgG (data not shown). The presence of PCR products in SRF and MKL1 precipitates indicates SRF and MKL1 binding to the CArG boxes of the tested genes. The binding appears in both unstimulated and BDNF-stimulated cortical neurons.

the Gapdh gene that does not contain any CArG box (Fig. 7). Hence, in intact neurons, MKL1 and SRF demonstrate an overlapping chromatin binding pattern as tested in several SRFregulated genes. Of those, at least $\operatorname{Srf}, \operatorname{JunB}$, and c-fos are strongly induced in cortical neurons by BDNF (Fig. $1 D, E$ ) (Arthur et al., 2004), further supporting the notion that MKL1 contributes to BDNF activation of SRF-driven transcription.

Induction of IEG, including c-fos, is a marker of neuronal activity that has been associated with neuronal plasticity (Kaczmarek and Chaudhuri, 1997; Valjent et al., 2001). Also, SRF was shown to contribute to neuronal induction of c-fos (Xia et al., 1996; Ramanan et al., 2005), whereas ChIP assay revealed SRF and MKL1 binding to the CArG box of the endogenous c-fos gene promoter (Fig. 7). Therefore, we also explored a possibility that MKL1 regulates the $\mathrm{c}-$ fos promoter. We used a reporter construct that contains TCF/SRF-regulated c-fos gene enhancer (nucleotides -356 to -297 ) fused to the minimal c-fos promoter (Wang and Prywes, 2000) (Fig. 8 A). Cortical neurons were cotransfected with the $c$-fos reporter plasmid together with DN-MKL1 or siMKL1. Forty-eight hours after transfection, neurons were stimulated for $8 \mathrm{~h}$ with BDNF $(10 \mathrm{ng} / \mathrm{ml})$, which resulted in c-fos reporter activation (Fig. $8 B, C$ ). This was significantly reduced by either DN-MKL1 or siMKL1 ( $p<0.001$ or 0.05 , respectively) (Fig. $8 B, C$ ). The $c$-fos enhancer contains a CArG box that is flanked by a TCF binding sequence enabling Elk-1/SRF cooperation during c-fos induction (Fig. $8 \mathrm{~A}$ ). However, BDNF activation of pm18, the mutated variant of c-fos enhancer reporter with inactivated TCF/Elk-1 binding site (Wang and Prywes, 2000) (Fig. $8 A$ ), was significantly decreased by DN-MKL1 or siMKL1 $(p<0.001)$ (Fig. 8D,E). These data suggest that in BDNFstimulated neurons, endogenous MKL1 activates SRF-driven transcription of c-fos reporter and that MKL1 regulation does not require TCF/Elk-1. 
A

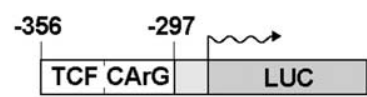

c-fos enhancer

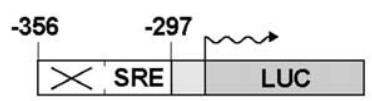

pm18

B
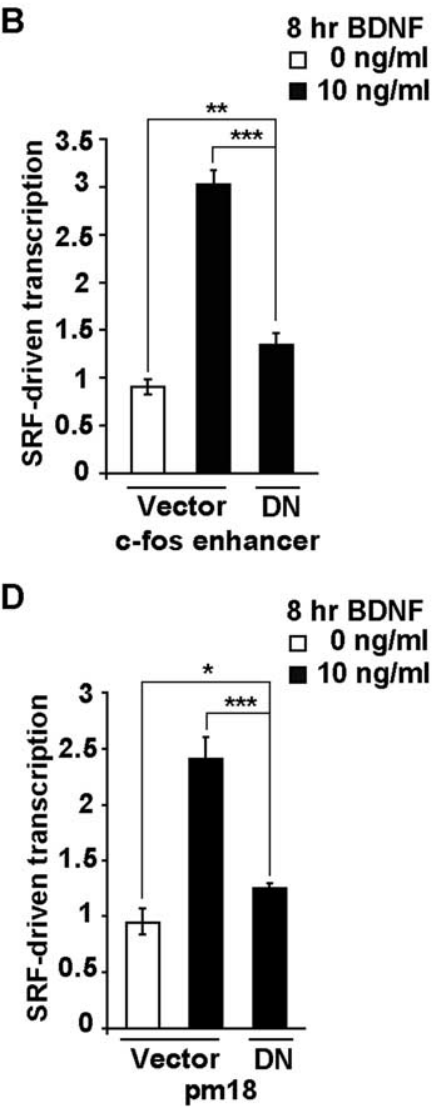

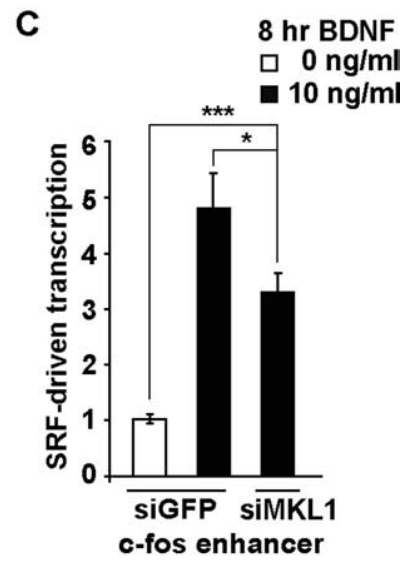

$E$

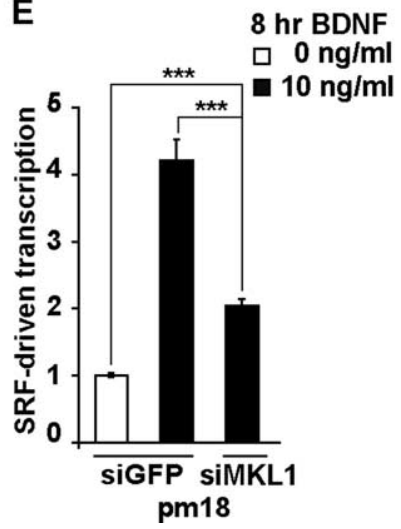

A

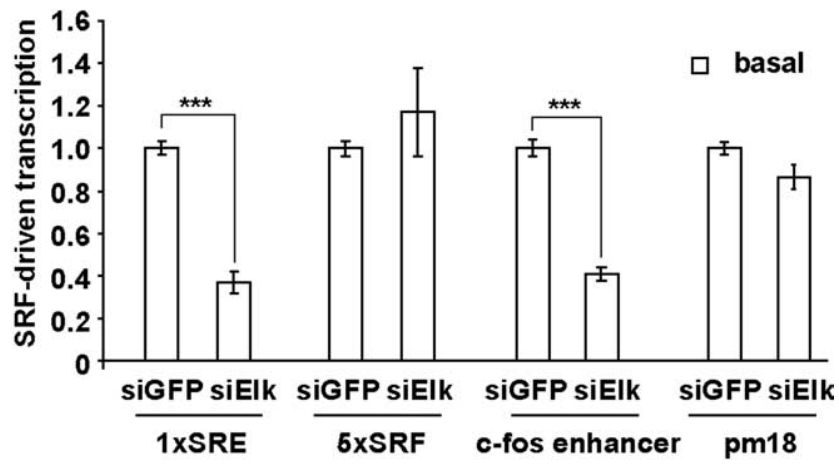

B

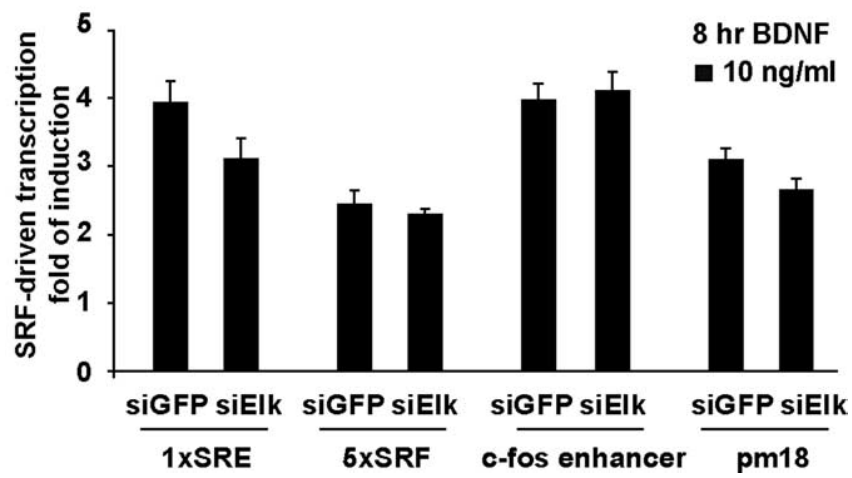

Figure 9. Inhibition of endogenous Elk-1 does not affect BDNF-mediated stimulation of SRF-driven transcription. Cortical neurons (4 DIV) were cotransfected with EF1 $\alpha$ LacZ together with the indicated reporter constructs and either a control siRNA (siGFP) or a 1:1 mix of two siRNA expression plasmids targeting Elk-1 (siElk-1; $0.1+0.1+1.0 \mu \mathrm{g}$ of plasmid DNA/5 $\times$ $10^{5}$ cells, respectively). Two days after transfection, cells were stimulated as indicated. $\boldsymbol{A}$, Effects of siElk-1 on basal SRF-driven transcription. SiElk-1 reduced basal transcription of 1XSRE and $c$-fos enhancer $\left.{ }^{* * *} p<0.001\right)$. B, SiElk-1 did not affect activation of SRF-driven transcription by BDNF. In $\boldsymbol{A}$ and $\boldsymbol{B}$, quadruplicate determinations from three independent experiments are depicted. Error bars indicate SEM.

transcription on 1xSRE, c-fos enhancer, 5xSRF, or pm18 reporters was not altered by siElk-1 (Fig. 9B). These data suggest that Elk-1 does not contribute to BDNF-mediated activation of SRF. Although we cannot rule out that other TCF family members are involved in BDNF signaling, MKL1 appears to be the first identified SRF coactivator linking BDNF to SRF activation.

\section{Neuronal MKL1 is regulated by ERK1/2}

ERK $1 / 2$ is an important transducer that activates gene expression programs in response to BDNF (Kaplan and Miller, 2000). Therefore, we tested whether ERK1/2 is required for MKL1 activation by BDNF. Forty-eight hours after cotransfection of the 5xSRF reporter construct together with the MKL1 expression plasmid, cortical neurons were stimulated for $8 \mathrm{~h}$ with $10 \mathrm{ng} / \mathrm{ml}$ BDNF in the presence or absence of an ERK1/2 pathway inhibitor, U0126 (50 $\mu \mathrm{M})$. BDNF activation of MKL1/SRF-driven transcription was abolished by U0126 (Fig. 10A). In contrast, inhibitors of either PI3K (30 $\mu \mathrm{M}$ LY294002) or ROCK (Rho-associated kinase; $10 \mu \mathrm{M}$ Y27632) did not affect MKL1/SRF activation by BDNF (Fig. 10A). A similar pattern of drug effects was observed when BDNF activation of MKL1 was monitored using the MKL1/ Gal4-SRF/E1B-luc reporter system (Fig. 10B). In addition, U0126 blocked BDNF activation of the 5xSRF reporter $(p<$ 0.001) (Fig. 10C). Finally, BDNF stimulation of the pm18 c-fos enhancer mutant, that because of an inactive TCF binding site is unable to respond to ERK1/2-stimulated Elk-1, was significantly 
A
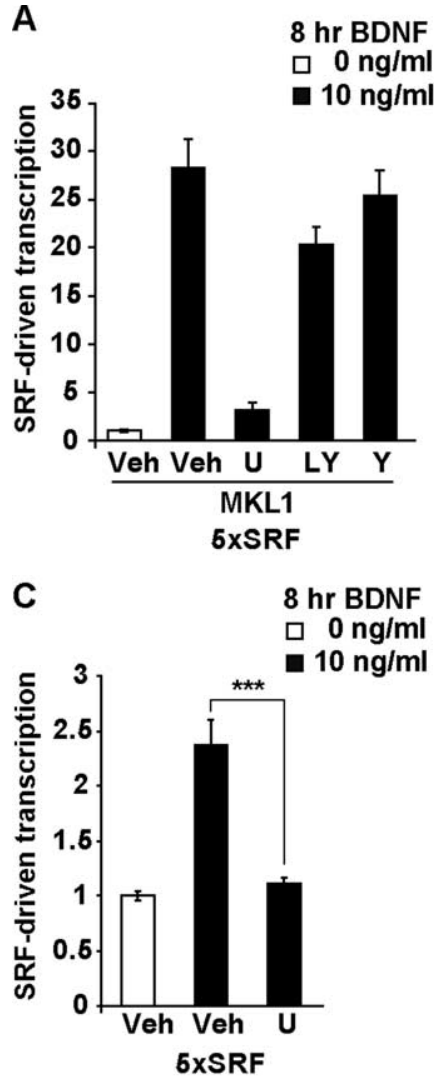

Figure 10. BDNF-mediated activation of MKL1 transcription requires ERK1/2 activity. Cortical neurons were transfected with the indicated constructs and other plasmids in $\boldsymbol{A}$ and $\boldsymbol{B}, \boldsymbol{C}$, or $\boldsymbol{D}$, as described for Figures 4,1 , or 7, respectively. BDNF stimulations were performed in the presence of drug inhibitors of the following pathways: ERK1/2 [50 $\mu \mathrm{m}$ U0126 (U)], PI3K [30 $\mu \mathrm{m}$ LY294002 (LY)], or Rho-associated kinase [10 $\mu \mathrm{M}$ Y27632 (Y)]. DMSO (0.2\%) was used as vehicle control (Veh). $\boldsymbol{A}, \boldsymbol{B}$, ERK1/2 inhibition abolished BDNF activation of SRF-driven transcription by the overexpressed MKL1. Data are quadruplicate determinations from representative experiments. Similar results were obtained in four independent experiments. C, D, ERK1/2 inhibition either abolished or reduced BDNF activation of $5 \mathrm{XSRF}$ or pm 18 reporters, respectively $\left({ }^{* *} p<\right.$ $0.01 ;{ }^{* * *} p<0.001$ ). Because BDNF effects on these reporters were mediated by endogenous MKL1 (see Figs. 5-7) and because neither 5xSRF nor pm18 has functional TCF sites to reflect ERK1/2-dependent activation of Elk-1, these data support the notion that endogenous MKL1 is regulated by the ERK1/2 pathway. Data represent quadruplicate determinations from three independent experiments. Error bars indicate SEM.

reduced by U0126 ( $p<0.01$ ) (Fig. 10D). Because endogenous MKL1 provides an important contribution to BDNF regulation of $5 \mathrm{xSRF}$ or pm 18 reporters (Figs. $5,6,8$ ), the ERK1/2 pathway appears to activate MKL1/SRF-driven transcription.

It was suggested that one of the mechanisms that control MKL1 activity is its nuclear translocation (Miralles et al., 2003). However, our results indicate a constitutive nuclear localization of MKL1 in cortical or hippocampal neurons (Figs. 2, 3). In addition, the nuclear pattern of MKL1 expression was not affected by BDNF or U0126 (data not shown). Therefore, it is unlikely that ERK1/2-mediated regulation of MKL1 is at the nuclear translocation step. Instead, it is possible that in stimulated neurons, activated ERK1/2 translocates to the nucleus and increases phosphorylation of nuclear MKL1 to turn on MKL1/SRF-driven transcription. This is supported by the observation that in fibroblasts, MKL1 was phosphorylated in an ERK1/2-dependent manner as determined by its shifting on SDS-PAGE (Miralles et al., 2003). Also, in cortical neurons that were stimulated with 10 $\mathrm{ng} / \mathrm{ml} \mathrm{BDNF}$ for $30 \mathrm{~min}$ or $8 \mathrm{~h}$, endogenous MKL1 displayed lowered electrophoretic mobility compared with unstimulated controls (Fig. 11A). ERK1/2 pathway inhibition with $\mathrm{U} 0126$ reduced that effect at $30 \mathrm{~min}$ while completely reversing it at $8 \mathrm{~h}$ (Fig. $11 \mathrm{~A}$ ). Therefore, BDNF triggers MKL1 phosphorylation, and the ERK1/2 pathway provides an important contribution to this effect. In addition, a $60 \mathrm{~min}$ inhibition of PP1- and PP2A-like phosphatases with $1 \mu \mathrm{M}$ okadaic acid triggered an electromobility shift of endogenous MKL1, further supporting the notion that in neurons, nuclear MKL1 is regulated by phosphorylation (Fig. 11A).

To test whether selective activation of the ERK1/2 pathway is sufficient to phosphorylate and activate MKL1, cortical neurons were cotransfected with expression plasmids for a constitutively active mutant form of the ERK1/2 activator MKK1 (CA-MKK1), wild-type ERK1, wild-type ERK2, and MKL1. Empty cloning vectors for MKK1 and ERK1/2 were used as controls (vectors). At $48 \mathrm{~h}$ after transfection of CA-MKK1/ERK1+ERK2, electromobility shift of MKL1 was observed (Fig. $11 \mathrm{~B}$ ). This indicates that selective activation ERK1/2 is sufficient to phosphorylate MKL1. The effect of ERK1/2 was specific as activation of the MEK5/ ERK5 or PI3K/Akt signaling pathways did not affect MKL1 phosphorylation. In addition, overexpression of CA-MKK1 but not CA-MEK5 or CA-PI3K (p110*) increased MKL1/SRF-driven transcription as determined by using the MKL1/Gal4-SRF/E1Bluc reporter system $(p<0.001)$ (Fig. 11C). Thus, the ERK1/2 pathway is both necessary and sufficient to phosphorylate MKL1 and activate MKL1/SRF-driven transcription.

ERK1/2 may affect transcription factors either directly or indirectly. For instance, ERK1/2-mediated phosphorylation activates Elk-1, whereas ERK1/2-dependent kinases phosphorylate and activate CREB (Impey et al., 1999; Adams and Sweatt, 2002). Therefore, we tested whether MKL1 is a suitable substrate for ERK2 in a test tube. Immunoprecipitated flag-MKL1 that was expressed in COS-7 cells was incubated with $\gamma^{32} \mathrm{P}$-ATP and active ERK2. This caused strong phosphorylation of MKL1 (Fig. $11 D$ ), which also resulted in an electromobility shift similar to that triggered by BDNF (Fig. $11 B, E$ ). Thus, direct ERK1/2mediated phosphorylation is a likely mechanism of MKL1 activation by BDNF.

\section{MKL1-dependent transcription is increased by}

synaptic activity

Synaptic activity is an important signal that activates pathways critical for neuronal plasticity. In cultured cortical neurons, pharmacological stimulation of synaptic activity has been shown to engage mechanisms similar to those underlying plasticity in vivo. For instance, combined treatment with a $\mathrm{GABA}_{\mathrm{A}}$ receptor antagonist, bicuculline, and a $\mathrm{K}^{+}$channel inhibitor, 4-AP, increased synaptic activity that, through stimulation of synaptic NMDA receptor (NMDAR), led to activation of ERK1/2, followed by an ERK1/2-dependent increase in CREB-regulated transcription (Hardingham et al., 2002; Lee et al., 2005). Therefore, we tested whether a bicuculline/4-AP-mediated increase in neuronal activity may also induce NMDAR- and ERK1/2-dependent activation of MKL1.

As a first step to evaluate this possibility, we determined bicuculline $(50 \mu \mathrm{M})$ plus 4 -AP $(2.5 \mathrm{~mm})$ effects on SRF-driven transcription. Eight-hour treatment with these agents increased the transcriptional activity of $1 \mathrm{xSRE}$ reporter 4.8 -fold over controls (Fig. 12A). The increase was abolished by the ERK1/2 pathway inhibitor U0126 $(50 \mu \mathrm{M})$ or a fast $\mathrm{Na}^{+}$channel blocker, TTX (1 $\mu \mathrm{M})$ (Fig. 12A). An NMDAR antagonist, MK801 (10 $\mu \mathrm{M})$, reduced the activation to 1.7 -fold of controls (Fig. 12A). In addi- 
A
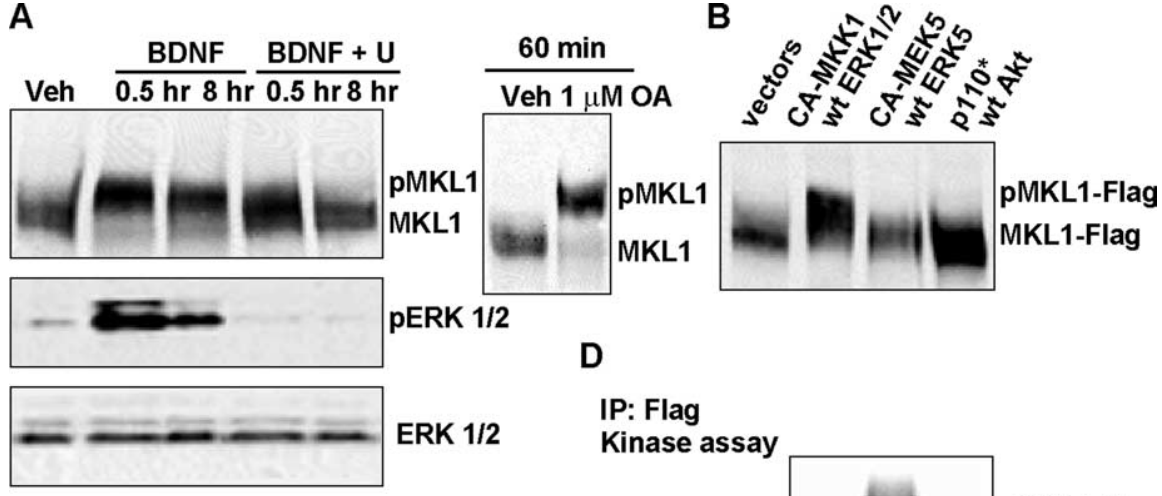

ERK 1/2

C

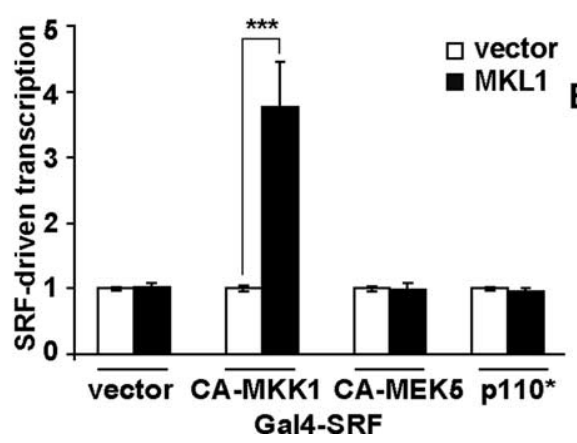

\section{IP: Flag}

Kinase assay

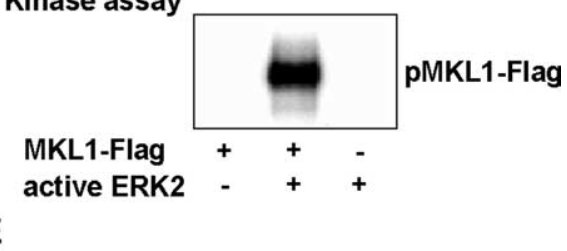

IP: Flag WB: MKL1

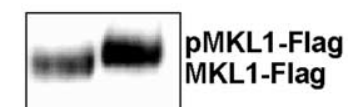

siGFP or siMKL1, respectively; $p<0.01$ ) (Fig. 12D). These data suggest that endogenous MKL1 contributes to activation of SRF-driven transcription by increased synaptic activity.

Finally, we evaluated whether transcriptional activity of an overexpressed MKL1 can be stimulated by synaptic activity. Cortical neurons were cotransfected with wild-type MKL1 expression plasmid and the 1xSRE reporter. After $48 \mathrm{~h}$, cells were stimulated with bicuculline/4-AP for $8 \mathrm{~h}$. This treatment increased 1xSRE activity 10.9-fold over control levels. The increase was abolished by U0126 or TTX, whereas it was partially reduced by MK801 (Fig. 12E). These data suggest that synaptic activity stimulated MKL1/SRF-driven transcription through the NMDARERK1/2 pathway. In support of this conclusion, we found that an $8 \mathrm{~h}$ stimulation of NMDAR with $20 \mu \mathrm{M}$ NMDA enhanced MKL1/SRF-driven transcription in MKL1overexpressing neurons (Fig. 12F). A similar response was noted after $8 \mathrm{~h}$ of $\mathrm{KCl}$-induced membrane depolarization that elevates $\left[\mathrm{Ca}^{2+}\right]_{\mathrm{i}}$ levels (Fig. 12F). Thus, MKL1 is activated by an increase in $\left[\mathrm{Ca}^{2+}\right]_{\mathrm{i}}$, which is the critical mediator for both NMDAR and synaptic activity signaling. Collectively, our data suggest that in addition to BDNF, synaptic activity regulates SRF-driven transcription by using MKL1 as an ERK1/2-activated transcriptional coactivator.

\section{Discussion}

Our data indicate the following: (1) BDNF activates SRF-driven transcription independently of TCF; (2) an alternative SRF regulator, MKL1, is expressed in forebrain neurons, including adult CA1 neurons, and contributes to BDNF-mediated regulation of SRF-driven transcription; (3) a likely mechanism underlying BDNF regulation of MKL1/SRF-driven transcription is ERK1/2-mediated phosphorylation of MKL1; and finally, (4) synaptic activity stimulates SRF-driven transcrip-

tion, bicuculline/4-AP treatment activated ERK1/2 (Fig. 12B). Similarly to the transcriptional effects, ERK1/2 activation was abolished by U0126 or TTX, whereas it was reduced by MK801 (Fig. 12B). Therefore, in cortical neurons, increased synaptic activity elevates SRF-driven transcription through a pathway that involves NMDAR-activated ERK1/2 signaling.

To test whether MKL1 contributes to activation of SRF-driven transcription by synaptic activity, we cotransfected cortical neurons with the 1xSRE reporter plasmid together with DN-MKL1 or siMKL1 or control plasmids. Two days after transfection, neurons were stimulated with bicuculline/4-AP for $8 \mathrm{~h}$. DN-MKL1 abolished 1xSRE activation by increased neuronal activity (4.3vs 1.3 -fold of unstimulated controls for empty vector or DNMKL1, respectively; $p<0.001$ ) (Fig. 12C). In addition, siMKL1 partially reduced $1 \mathrm{xSRE}$ activation in bicuculline/4-APstimulated neurons (3.5- vs 2.1-fold of unstimulated controls for tion, in part through the NMDAR-ERK1/2-MKL1 pathway. Our data identify MKL1 as a novel link between ERK1/2 and SRF. They also raise an interesting possibility that MKL1 plays a role in SRF-regulated gene expression programs that contribute to neuronal plasticity.

We observed that in cortical neurons, BDNF activates SRFdriven transcription, which is mediated by promoters containing CArG boxes but not TCF binding sites. In addition, BDNF increased expression of SRF, the promoter of which is regulated by SRF but not TCF/SRF (Spencer and Misra, 1996; Miano, 2003). Thus, BDNF regulation of SRF-driven transcription occurs without interactions of SRF with TCF, indicating an involvement of TCF-independent mechanisms. This is important because it suggests that in addition to genes controlled by TCF/CArG elements, BDNF may also induce genes 

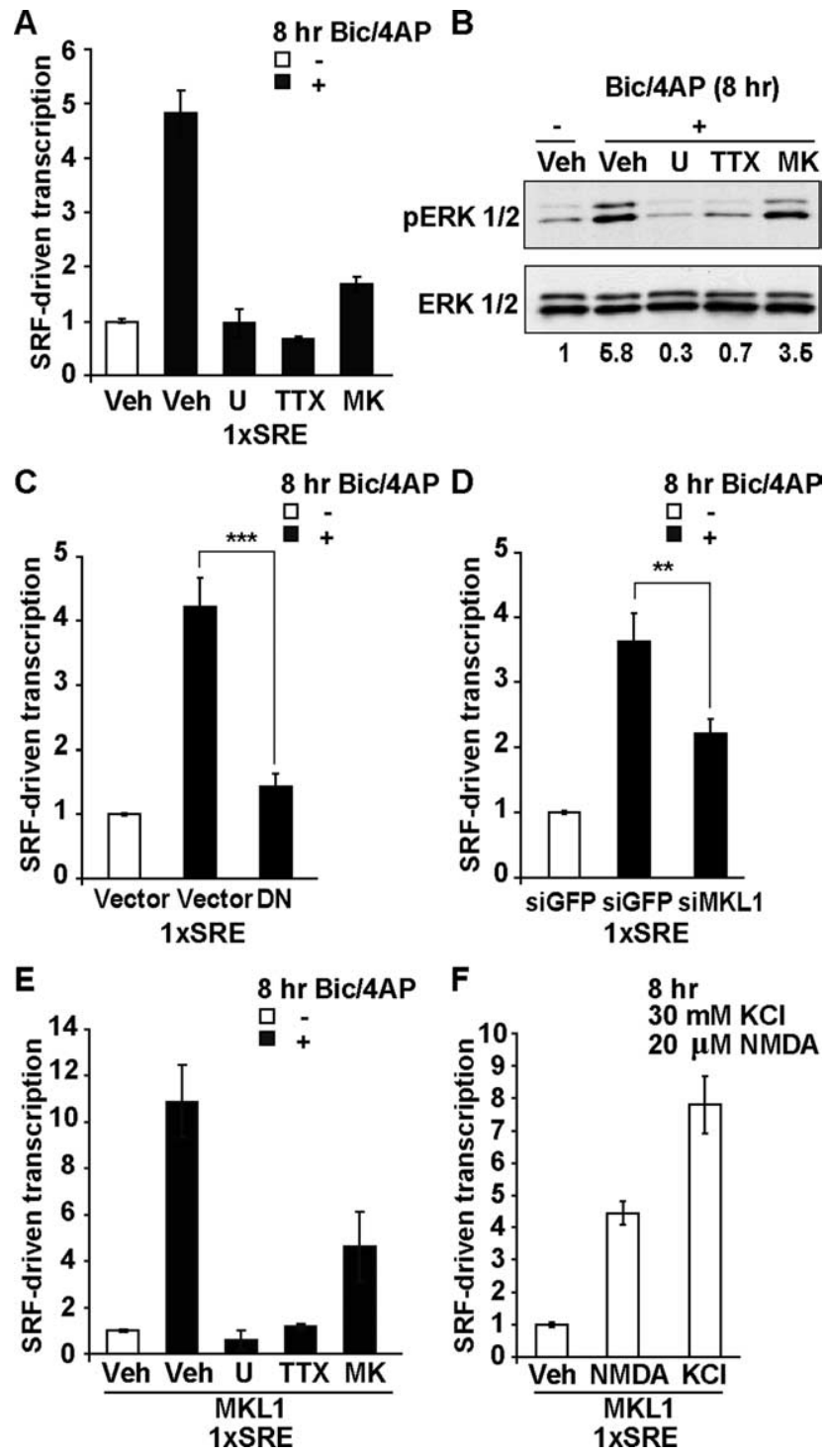

Figure 12. Synaptic activity stimulated MKL1/SRF-driven transcription. Cortical neurons (5 DIV) were transfected with the indicated constructs in $A, C, D$, or $\boldsymbol{E}$ and $\boldsymbol{F}$, as described for Figures $1,5,6$, or 4 , respectively. After $48 \mathrm{~h}$, cells were stimulated with $50 \mu \mathrm{m}$ bicuculline and $2.5 \mathrm{~mm}$ 4-AP (Bic/4-AP), NMDA, or KCl. The indicated stimulations were done in the presence of vehicle (Veh; 0.2\% DMSO) or $50 \mu \mathrm{m}$ U0126 (U), $1 \mu \mathrm{m}$ TTX, or $10 \mu \mathrm{m}$ MK801 (MK). Untransfected cortical neurons were used in $\boldsymbol{B}$. $\boldsymbol{A}, \mathrm{Bic} / 4 \mathrm{AP}$ activated the 1XSRE reporter in a TTX-sensitive manner, suggesting that increased synaptic activity regulates SRF-driven transcription. The activation required the ERK1/2 pathway and NMDAR. Means of quadruplicate determinations \pm SEM are depicted; similar results were obtained in three independent experiments. $\boldsymbol{B}$, Cortical neurons (7 DIV) were stimulated as in $\boldsymbol{A}$. The numbers under the blot indicate the relative levels of pERK1/2 after normalization against total ERK1/2. Increased synaptic activity stimulated ERK1/2, at least in part, via NMDAR. C, D, Effects of inhibiting endogenous MKL1 on synaptic activity-mediated increase in SRF-driven transcription. The increase in SRF-driven transcription was abolished with DN-MKL1 or reduced with siMKL1 $\left({ }^{* * *} p<0.001\right.$ and ${ }^{* *} p<0.01$, respectively). Data represent quadruplicate determinations from three independent experiments. $\boldsymbol{E}$, Effects of synaptic activity on SRF-driven transcription by the overexpressed MKL1. Bic/4-AP increased transcriptional activity of the overexpressed MKL1. The increase was abolished by U0126 or TTX and partially reduced by MK801. F, Effects of NMDA or KCl on SRF-driven transcription by the overexpressed MKL1. Transcriptional activity of the overexpressed MKL1 was activated by NMDA or KCl treatments. In $\boldsymbol{E}$ and $\boldsymbol{F}$, the means of quadruplicate determinations \pm SEM are depicted; similar results were obtained in three independent experiments.

that are under the control of promoters regulated by CArG boxes only. Of note, a TCF-independent mechanism of SRF activation was also suggested in PC12 cells stimulated with another member of the neurotrophin family, NGF (Bonni et al., 1995; Poser et al., 2000). Hence, neurotrophins might activate a novel pathway that does not require the TCF binding site but involves members of the myocardin family of transcriptional coactivators. Obviously, this does not exclude a possibility that TCFs contribute to SRF-driven transcriptional responses activated by neurotrophins.

We found that a member of the myocardin family, MKL1, is expressed in rodent forebrain neurons and contributes to the stimulation of SRF-driven transcription by BDNF as well as synaptic activity. Previous studies reported MKL1 mRNA expression in the adult mouse brain and the presence of MKL1 protein in rat embryonic cortical neurons in culture (Wang and Prywes, 2000; Sasazuki et al., 2002; Tabuchi et al., 2005). Our study extends these observations by demonstrating MKL1 expression in adult brain regions that undergo neuronal plasticity and by identifying neuron-specific signals that activate MKL1.

Our results identify MKL1 as a novel target for ERK1/2mediated regulation of SRF-driven transcription in neurons. SRF regulation by ERK1/2 is well established (for review, see Shaw and Saxton, 2003) and was demonstrated in cultured primary neurons responding to BDNF (Chang et al., 2004). The major mechanism mediating that effect has been proposed to rely on ERK1/ 2-mediated phosphorylation of the p62TCF/Elk-1 (Shaw and Saxton, 2003). However, in neurons, the existence of alternative mechanisms linking SRF to ERK1/2 is possible. This is supported by the moderate effects of Elk-1 knock-out on neuronal activitymediated induction of IEG (Cesari et al., 2004). Conversely, neuronal activity did not trigger IEG expression if SRF or ERK1/2 were inhibited (Sgambato et al., 1998b; Valjent et al., 2001; Ramanan et al., 2005). Our results indicate that MKL1 contributes to this alternative coupling between ERK1/2 and SRF.

Our data indicate that MKL1 but not Elk-1 is required for SRF activation by BDNF. However, the relative contribution of MKL1, Elk-1, and other SRF coactivators to the neurotrophinactivated gene expression programs remains to be determined. In smooth muscle cells, the ERK1/2-activated Elk-1 antagonized myocardin-mediated transcription by competing for SRF binding (Wang et al., 2004). Moreover, it has been proposed that in this system, the main function of Elk-1 is not activation of gene expression but transcriptional repression of myocardin-activated SRF (Wang et al., 2004). It remains to be tested whether neuronal ERK1/2 can engage a similar Elk-1-mediated feedback to regulate MKL1 function at endogenous promoters.

Our data indicate that in cultured neurons, endogenous MKL1 is constitutively localized to the nucleus with no detectable presence in other cellular compartments. Neither BDNF nor U0126 altered that nuclear pattern of MKL1 expression (data not shown). In addition, in adult rodent brain, we found nuclear localization of MKL1 in the hippocampal CA1 subfield and deep layers of the neocortex. Similar localization of MKL1 was reported in primary smooth muscle cells (Du et al., 2004). In contrast, in fibroblasts, MKL1 was localized in the cytosol and relocated to the nucleus after serum stimulation (Miralles et al., 2003; Du et al., 2004). This has been proposed to be mediated by its selective association with cytosolic G-actin that, because of serum-stimulated RhoA activity, is turned into F-actin, thereby releasing MKL1 and enabling its translocation to the nucleus (Miralles et al., 2003). Similar nuclear transclocation was observed in primary cortical neurons isolated from rat embryos that were transfected with constructs stimulating RhoA signaling ( $\mathrm{Ta}-$ buchi et al., 2005). Hence, postnatal neurons that were used in our study may have switched to a different mode of MKL1 regu- 
lation by ERK1/2-mediated phosphorylation of MKL1 that is already present in the nucleus.

Our results strongly support the critical role of ERK1/2 in MKL1/SRF activation by BDNF or increased synaptic activity. In addition to ERK1/2, SRF activity may be regulated by PI3K. PI3K contributed to TCF-independent activation of SRF in NGFstimulated PC12 cells and BDNF activation of an SRE reporter in cultured cortical neurons (Poser et al., 2000; Chang et al., 2004). In our hands, PI3K was neither required nor sufficient to activate MKL1/SRF-driven transcription. Hence, PI3K may regulate SRF activity that is independent of MKL1 or contribute to MKL1 activation in a stimulus/cell type-specific manner. Several studies demonstrated that RhoA is an important regulator of MKL1mediated SRF-driven transcription (Cen et al., 2003; Miralles et al., 2003; Du et al., 2004; Philippar et al., 2004; Tabuchi et al., 2005). The contribution of RhoA to BDNF- or synaptic activitymediated stimulation of MKL1/SRF-driven transcription is unclear. In our hands, overexpression of a RhoA inhibitor, C3 transferase, induced neurotoxicity, making transcription studies difficult (K. Kalita and M. Hetman, unpublished observation). Nevertheless, the constitutively nuclear localization of MKL1 in postnatal forebrain neurons suggests that, at least in this system, the RhoA-stimulated nuclear targeting of MKL1 is not involved in BDNF- or synaptic activity-induced MKL1/SRF-driven transcription.

The observation that MKL1/SRF is activated by increased synaptic activity widens the spectrum of transcriptional regulators that respond to synaptic transmission. In cultured forebrain neurons, synaptic activity-coupled transcription requires calcium signaling that is activated by L-type voltage-gated calcium channels (L-VGCCs) and/or NMDAR (Graef et al., 1999; Hardingham et al., 2002). Indeed, blocking NMDAR reduced MKL1/SRF response to increased synaptic activity. Because the reduction was not complete, additional input from L-VGCC is a likely regulator of MKL1/SRF. In PC12 cells, L-VGCC activation by $\mathrm{KCl}$ stimulated SRF transcription in the absence of a TCFbinding site, further supporting the possibility that MKL1 or MKL1-like coactivators of SRF are regulated by calcium signaling (Misra et al., 1994).

The functional consequences of MKL1/SRF-driven transcription in neurons are unclear. In cultured mouse hippocampal neurons, overexpression of DN-MKL1 mutants impaired both neurite outgrowth and ephrin-A5-induced growth cone collapse (Knoll et al., 2006). These effects were not observed in SRFdeficient neurons, whereas SRF-deficient mice displayed severe disturbances in targeting of dentate gyrus axons to CA3 pyramidal neurons (Knoll et al., 2006). In addition, genes encoding SRF, actin, and axonal guidance molecules including ephrins A4, A7, and semaphorin $3 \mathrm{C}$ were identified as candidate targets for SRF (Knoll et al., 2006). Therefore, it was proposed that MKL1 and SRF coordinate a gene-expression program that is critical for neurite elongation and guidance. Neurotrophins, including BDNF, provide an important signal for neurite outgrowth during development (Markus et al., 2002). Because MKL1/SRF-driven transcription is activated by BDNF, it is tempting to speculate that MKL1 contributes to BDNF-induced neurite outgrowth by stimulating elongation while ensuring responsiveness to the repulsive cues mediated by the ephrin/semaphorin systems.

The emerging role of SRF in adult brain plasticity suggests potential involvement of MKL1 in learning and memory (Dash et al., 2005; Ramanan et al., 2005; Etkin et al., 2006). This is supported by the presence of MKL1 in the adult hippocampal CA1 region, which shows a high level of neuronal plasticity (Bliss and
Collingridge, 1993). In addition, MKL1/SRF-driven transcription is increased by synaptic activity, which is a critical switch for adult neuronal plasticity (Bliss and Collingridge, 1993). Finally, we show that MKL1 is regulated by stimulation of NMDAR and activation of ERK1/2, which are both required in many plasticity paradigms (Bliss and Collingridge, 1993; Impey et al., 1999; Adams and Sweatt, 2002). However, the role of MKL1 in SRF-driven gene expression programs underlying adult plasticity remains to be determined.

In summary, we identified MKL1 as a novel coactivator of SRF that, in forebrain neurons, contributes to SRF-driven transcription in response to BDNF or synaptic activity. In addition, MKL1 appears to be a new target for ERK1/2 signaling, providing a TCF-independent link between ERK1/2 and SRF. Therefore, MKL1 is a likely regulator of SRF-dependent processes in brain development and/or adult brain plasticity.

\section{References}

Adams JP, Sweatt JD (2002) Molecular psychology: roles for the ERK MAP kinase cascade in memory. Annu Rev Pharmacol Toxicol 42:135-163.

Arthur JS, Fong AL, Dwyer JM, Davare M, Reese E, Obrietan K, Impey S (2004) Mitogen- and stress-activated protein kinase 1 mediates cAMP response element-binding protein phosphorylation and activation by neurotrophins. J Neurosci 24:4324-4332.

Bliss TVP, Collingridge GL (1993) A synaptic model of memory: long-term potentiation in the hippocampus. Nature 361:31-39.

Bonni A, Ginty DD, Dudek H, Greenberg ME (1995) Serine 133phosphorylated CREB induces transcription via a cooperative mechanism that may confer specificity to neurotrophin signals. Mol Cell Neurosci 6:168-183.

Brummelkamp TR, Bernards R, Agami R (2002) A system for stable expression of short interfering RNAs in mammalian cells. Science 296:550-553.

Cavanaugh JE, Ham J, Hetman M, Poser S, Yan C, Xia Z (2001) Differential regulation of mitogen-activated protein kinases ERK1/2 and ERK5 by neurotrophins, neuronal activity, and cAMP in neurons. J Neurosci 21:434-443.

Cen B, Selvaraj A, Burgess RC, Hitzler JK, Ma Z, Morris SW, Prywes R (2003) Megakaryoblastic leukemia 1, a potent transcriptional coactivator for serum response factor (SRF), is required for serum induction of SRF target genes. Mol Cell Biol 23:6597-6608.

Cen B, Selvaraj A, Prywes R (2004) Myocardin/MKL family of SRF coactivators: key regulators of immediate early and muscle specific gene expression. J Cell Biochem 93:74-82.

Cesari F, Rennekampff V, Vintersten K, Vuong LG, Seibler J, Bode J, Wiebel FF, Nordheim A (2004) Elk-1 knock-out mice engineered by Flp recombinase-mediated cassette exchange. Genesis 38:87-92.

Chang SH, Poser S, Xia Z (2004) A novel role for serum response factor in neuronal survival. J Neurosci 24:2277-2285.

Cherrington JM, Mocarski ES (1989) Human cytomegalovirus iel transactivates the a promoter-enhancer via an 18-base-pair repeat element. J Virol 63:1435-1440.

Dash PK, Orsi SA, Moore AN (2005) Sequestration of serum response factor in the hippocampus impairs long-term spatial memory. J Neurochem 93:269-278.

Du KL, Chen M, Li J, Lepore JJ, Mericko P, Parmacek MS (2004) Megakaryoblastic leukemia factor-1 transduces cytoskeletal signals and induces smooth muscle cell differentiation from undifferentiated embryonic stem cells. J Biol Chem 279:17578-17586.

Dudek H, Datta SR, Franke TF, Birnbaum MJ, Yao RJ, Cooper GM, Segal RA, Kaplan DR, Greenberg ME (1997) Regulation of neuronal survival by the serine-threonine protein kinase Akt. Science 275:661-665.

Etkin A, Alarcon JM, Weisberg SP, Touzani K, Huang YY, Nordheim A, Kandel ER (2006) A role in learning for SRF: deletion in the adult forebrain disrupts LTD and the formation of an immediate memory of a novel context. Neuron 50:127-143.

Frost JA, Steen H, Shapiro P, Lewis T, Ahn N, Shaw PE, Cobb MH (1997) Cross-cascade activation of ERKs and ternary complex factors by Rho family proteins. EMBO J 16:6426-6438.

Graef IA, Mermelstein PG, Stankunas K, Neilson JR, Deisseroth K, Tsien RW, 
Crabtree GR (1999) L-type calcium channels and GSK-3 regulate the activity of NF-ATc4 in hippocampal neurons. Nature 401:703-708.

Habas A, Kharebava G, Szatmari E, Hetman M (2006) NMDA neuroprotection against a phosphatidylinositol-3 kinase inhibitor, LY294002 by NR2B-mediated suppression of glycogen synthase kinase-3beta-induced apoptosis. J Neurochem 96:335-348.

Hanlon M, Sealy L (1999) Ras regulates the association of serum response factor and CCAAT/enhancer-binding protein beta. J Biol Chem 274:14224-14228.

Hardingham GE, Fukunaga Y, Bading H (2002) Extrasynaptic NMDARs oppose synaptic NMDARs by triggering CREB shut-off and cell death pathways. Nat Neurosci 5:405-414.

Hetman M, Kanning K, Smith-Cavanaugh JE, Xia Z (1999) Neuroprotection by brain-derived neurotrophic factor is mediated by extracellularsignal-regulated kinase and phosphatidylinositol-3 kinase. J Biol Chem 274:22569-22580.

Hetman M, Hsuan SL, Habas A, Higgins MJ, Xia Z (2002) ERK1/2 antagonizes glycogen synthase kinase-3beta-induced apoptosis in cortical neurons. J Biol Chem 277:49577-49584.

Hu Q, Klippel A, Muslin AJ, Fantl WJ, Williams LT (1995) Ras-dependent induction of cellular responses by constitutively active phosphatidylinositol-3 kinase. Science 268:100-102.

Impey S, Obrietan K, Wong ST, Poser S, Yano S, Wayman G, Deloulme JC, Chan G, Storm DR (1998) Crosstalk between ERK and PKA is required for $\mathrm{Ca} 2+$ stimulation of CREB-dependent transcription and ERK nuclear translocation. Neuron 21:869-883.

Impey S, Obrietan K, Storm DR (1999) Making new connections: role of ERK/MAP kinase signaling in neuronal plasticity. Neuron 23:11-14.

Kaczmarek L, Chaudhuri A (1997) Sensory regulation of immediate-early gene expression in mammalian visual cortex: implications for functional mapping and neural plasticity. Brain Res Brain Res Rev 23:237-256.

Kaplan DR, Miller FD (2000) Neurotrophin signal transduction in the nervous system. Curr Opin Neurobiol 10:381-391.

Knoll B, Kretz O, Fiedler C, Alberti S, Schutz G, Frotscher M, Nordheim A (2006) Serum response factor controls neuronal circuit assembly in the hippocampus. Nat Neurosci 9:195-204.

Lee B, Butcher GQ, Hoyt KR, Impey S, Obrietan K (2005) Activitydependent neuroprotection and cAMP response element-binding protein (CREB): kinase coupling, stimulus intensity, and temporal regulation of CREB phosphorylation at serine 133. J Neurosci 25:1137-1148.

Lonze BE, Ginty DD (2002) Function and regulation of CREB family transcription factors in the nervous system. Neuron 35:605-623.

Mansour SJ, Matten WT, Hermann AS, Candia JM, Rong S, Fukasawa K, Vande Woude GF, Ahn NG (1994) Transformation of mammalian cells by constitutively active MAP kinase kinase. Science 265:966-970.

Markus A, Patel TD, Snider WD (2002) Neurotrophic factors and axonal growth. Curr Opin Neurobiol 12:523-531.

Miano JM (2003) Serum response factor: toggling between disparate programs of gene expression. J Mol Cell Cardiol 35:577-593.

Miralles F, Posern G, Zaromytidou AI, Treisman R (2003) Actin dynamics control SRF activity by regulation of its coactivator MAL. Cell 113:329-342.

Misra RP, Bonni A, Miranti CK, Rivera VM, Sheng M, Greenberg ME (1994) Calcium entry through L-type voltage sensitive calcium channels can activate transcription via the serum response factor. J Biol Chem 269:25483-25493.

Morris TA, Jafari N, Rice AC, Vasconcelos O, DeLorenzo RJ (1999) Persistent increased DNA-binding and expression of serum response factor occur with epilepsy-associated long-term plasticity changes. J Neurosci 19:8234-8243

Philippar U, Schratt G, Dieterich C, Muller JM, Galgoczy P, Engel FB, Keating
MT, Gertler F, Schule R, Vingron M, Nordheim A (2004) The SRF target gene Fhl 2 antagonizes RhoA/MAL-dependent activation of SRF. Mol Cell $16: 867-880$.

Poser S, Impey S, Trinh K, Xia Z, Storm DR (2000) SRF-dependent gene expression is required for PI3-kinase-regulated cell proliferation. EMBO J 19:4955-4966.

Ramanan N, Shen Y, Sarsfield S, Lemberger T, Schutz G, Linden DJ, Ginty DD (2005) SRF mediates activity-induced gene expression and synaptic plasticity but not neuronal viability. Nat Neurosci 8:759-767.

Sasazuki T, Sawada T, Sakon S, Kitamura T, Kishi T, Okazaki T, Katano M, Tanaka M, Watanabe M, Yagita H, Okumura K, Nakano H (2002) Identification of a novel transcriptional activator, BSAC, by a functional cloning to inhibit tumor necrosis factor-induced cell death. J Biol Chem 277:28853-28860.

Selvaraj A, Prywes R (2004) Expression profiling of serum inducible genes identifies a subset of SRF target genes that are MKL dependent. BMC Mol Biol 5:13.

Sgambato V, Vanhoutte P, Pages C, Rogard M, Hipskind R, Besson MJ, Caboche J (1998a) In vivo expression and regulation of Elk-1, a target of the extracellular-regulated kinase signaling pathway, in the adult rat brain. J Neurosci 18:214-226.

Sgambato V, Pages C, Rogard M, Besson MJ, Caboche J (1998b) Extracellular signal-regulated kinase (ERK) controls immediate early gene induction on corticostriatal stimulation. J Neurosci 18:8814-8825

Shaw PE, Saxton J (2003) Ternary complex factors: prime nuclear targets for mitogen-activated protein kinases. Int J Biochem Cell Biol 35:1210-1226.

Spencer JA, Misra RP (1996) Expression of the serum response factor gene is regulated by serum response factor binding sites. J Biol Chem 271:16535-16543.

Tabuchi A, Estevez M, Henderson JA, Marx R, Shiota J, Nakano H, Baraban JM (2005) Nuclear translocation of the SRF co-activator MAL in cortical neurons: role of RhoA signalling. J Neurochem 94:169-180.

Valjent E, Caboche J, Vanhoutte P (2001) Mitogen-activated protein kinase/extracellular signal-regulated kinase induced gene regulation in brain: a molecular substrate for learning and memory? Mol Neurobiol 23:83-99.

Vanhoutte P, Barnier JV, Guibert B, Pages C, Besson MJ, Hipskind RA, Caboche J (1999) Glutamate induces phosphorylation of Elk-1 and CREB, along with c-fos activation, via an extracellular signal-regulated kinasedependent pathway in brain slices. Mol Cell Biol 19:136-146.

Wang DZ, Olson EN (2004) Control of smooth muscle development by the myocardin family of transcriptional coactivators. Curr Opin Genet Dev 14:558-566.

Wang Y, Prywes R (2000) Activation of the c-fos enhancer by the erk MAP kinase pathway through two sequence elements: the c-fos AP-1 and p62TCF sites. Oncogene 19:1379-1385.

Wang Z, Wang DZ, Hockemeyer D, McAnally J, Nordheim A, Olson EN (2004) Myocardin and ternary complex factors compete for SRF to control smooth muscle gene expression. Nature 428:185-189.

Xia Z, Dudek H, Miranti CK, Greenberg ME (1996) Calcium influx via the NMDA receptor induces immediate early gene transcription by a MAP kinase/ERK-dependent mechanism. J Neurosci 16:5425-5436.

Yang P, Baker KA, Hagg T (2005) A disintegrin and metalloprotease 21 (ADAM21) is associated with neurogenesis and axonal growth in developing and adult rodent CNS. J Comp Neurol 490:163-179.

Zaromytidou AI, Miralles F, Treisman R (2006) MAL and ternary complex factor use different mechanisms to contact a common surface on the serum response factor DNA-binding domain. Mol Cell Biol 26:41344148. 